\title{
Long Noncoding RNAs in Digestive System Malignancies: A Novel Class of Cancer Biomarkers and Therapeutic Targets?
}

\author{
Athina Kladi-Skandali, Kleita Michaelidou, Andreas Scorilas, and Konstantinos Mavridis \\ Department of Biochemistry and Molecular Biology, University of Athens, 15701 Athens, Greece \\ Correspondence should be addressed to Konstantinos Mavridis; mavridiskos@gmail.com
}

Received 23 November 2014; Accepted 20 April 2015

Academic Editor: Roberto César P. Lima-Júnior

Copyright ( 2015 Athina Kladi-Skandali et al. This is an open access article distributed under the Creative Commons Attribution License, which permits unrestricted use, distribution, and reproduction in any medium, provided the original work is properly cited.

High throughput methodologies have revealed the existence of an unexpectedly large number of long noncoding RNAs (lncRNAs). The unconventional role of lncRNAs in gene expression regulation and their broad implication in oncogenic and tumor suppressive pathways have introduced lncRNAs as novel biological tumor markers. The most prominent example of lncRNAs application in routine clinical practice is PCA3, a FDA-approved biomarker for prostate cancer. Regarding digestive system malignancies, the oncogenic HOTAIR is one of the most widely studied lncRNAs in the preclinical level and has already been identified as a potent prognostic marker for major malignancies of the gastrointestinal tract. Here, we provide an overview of recent findings regarding the emerging role of lncRNAs not only as key regulators of cancer initiation and progression in colon, stomach, pancreatic, liver, and esophageal cancers, but also as reliable tumor markers and therapeutic tools. IncRNAs can be easily, rapidly, and cost-effectively determined in tissues, serum, and gastric juice, making them highly versatile analytes. Taking also into consideration the largely unmet clinical need for early diagnosis and more accurate prognostic/predictive markers for gastrointestinal cancer patients, we comment upon the perspectives of lncRNAs as efficient molecular tools that could aid in the clinical management.

\section{Introduction}

Gastrointestinal (GI) cancer is an umbrella term which refers to a diverse group of tumors that affect the digestive system and accessory organs, including the esophagus, stomach, gallbladder, liver, pancreas, and small and large intestine [1]. According to recent GLOBOCAN data, 4.06 million new cases and 3.03 million deaths of GI cancer occurred in 2012, worldwide. Liver (LC), gastric (GC), and colorectal (CRC) cancers are ranked second to fourth in terms of worldwide cancer-related mortality, behind only lung cancer [2]. Pancreatic cancer incidence is increasing rapidly and prognosis remains poor, with a 5-year survival rate of as little as $6 \%$ [3]. Esophageal cancer is a virulent malignancy of the upper GI tract and overall 5-year relative survival remains below 20\% [4].

One of the common features of digestive system carcinomas, which can partially explain the abovementioned dismal statistics, is the lack of early alarming clinical symptoms.
Early neoplastic lesions are macroscopically similar to normal tissues and this complicates their detection with endoscopic and/or imaging approaches [1]. Adding to this perplexity, robust diagnostic biomarkers for most GI cancers are not yet available. For instance, even serum carbohydrate antigen 19-9 (CA19-9) has relatively poor specificity and sensitivity, to warrant its use as a screening biomarker for pancreatic cancer $[1$, 5]. Furthermore, a large proportion of GI cancer patients who have undergone treatment, suffer from frequent relapse and metastatic recurrence of disease. Unpredictable chemotherapy resistance is another major contributor to the poor clinical outcome of advanced stage GI cancer patients [6-8].

An in-depth understanding of the previously uncharted molecular pathways that promote the multistep process of GI cancer initiation, progression, and chemoresistance could be effectively translated into biomarkers that will accelerate the realization of an optimal clinical management for GI cancer patients. One such molecular mechanism, as suggested by continuously growing body of evidence, is the regulation 
by long noncoding RNAs (lncRNAs), a species of RNA molecules that are steadily becoming the next frontier for cancer translational research.

\section{Long Noncoding RNAs: An Unconventionally Unique Component of the Human Transcriptome}

The application of high-throughput sequencing technologies and bioinformatic methods, uncovered the existence of an extraordinary number of non-protein coding RNAs (ncRNAs) expressed by the human genome [9]. These untranslated RNA transcripts are categorized into short and long ncRNAs according to their size, which in turn, can be divided into subclasses based on their functional and structural features [10]. Although research interest has long been monopolized by short ncRNAs, such as microRNAs, a lot of attention is now focused towards the abundant and diverse class of lncRNAs.

lncRNAs are endogenous cellular RNA molecules more than 200 nucleotides long, which do not possess open reading frames (ORFs) of significant length. Although compelling evidence shows that lncRNAs lack protein-coding capacity, it is possible that a subset of IncRNAs with small ORFs, may indeed code for short peptides [11, 12]. lncRNAs are predominantly transcribed by RNA polymerase II and are subsequently subjected to $5^{\prime}$-end capping, $3^{\prime}$-end polyadenylation, and splicing. However, multiple lncRNAs are produced by RNA polymerase III and remain nonpolyadenylated [13]. IncRNAs are localized to the nucleus and cytoplasm where they can interact with DNA, RNA, and proteins and act as gene expression regulators at the transcriptional and/or posttranscriptional levels. Moreover, IncRNAs display tissue-, cell-, developmental-, and disease-specific expression patterns [14] and are detectable in several bodily fluids, such as blood and urine [15].

Recently, Ma et al. [16] thoroughly devised lncRNAs according to their genomic location, their distance of the target gene, the targeting mechanisms, and their exact mode of action. Based on their genomic position, lncRNAs are characterized as sense, antisense, intronic, and intergenic lncRNAs (lincRNAs). A lncRNA molecule can be either a cis-acting agent, regulating the expression of a neighbor gene of the same chromosome, or a trans-acting element, inducing epigenetic changes on a distant gene of the same or different chromosome. In terms of targeting mechanisms, lncRNAs act as (1) markers for the spatiotemporal expression of the target genes (signal), (2) adaptors for the assembling of functional protein complexes (scaffold), (3) factors that tether specific proteins and determine the proper localization of the resultant complex (guide), and (4) molecules that prevent the interaction of another RNA or protein with its natural targets (decoy). Based on their mechanism of action, lncRNAs are categorized into three subgroups that regulate gene transcription, posttranscriptional events, and other procedures such as RNA interference [16].

\section{Noncoding Does Not Mean Nonfunctional: Mechanisms of Action of lncRNAs and Their Role in Physiology}

The first human lncRNA gene, H19, was cloned and sequenced by Brannan et al., in 1990 [17]. A year later, the $\mathrm{X}$-inactive-specific transcript (XIST) was identified [18]. Currently, the estimated number of $\operatorname{lncRNAs}$ genes in the human genome is 56018 [19], the exact functions of which are only beginning to be unraveled. lncRNAs are considered as ideal orchestrators of RNA-based regulatory mechanisms, adding a new layer to the, already complex, network of gene expression control. Although information regarding the exact mechanisms of action of lncRNA remain scant, mounting experimental data suggest that they are involved in each step of the gene information processing, via distinct mechanisms [20].

lncRNAs play crucial roles in epigenetic regulation of gene expression. In this case, cis- or trans-acting $\operatorname{lncR}$ NAs (e.g., XIST and HOTAIR, resp.), serving as scaffold molecules, direct the recruitment of histone or chromatin modifying complexes to the target gene and induce its repression. Besides, activating lncRNAs, known as enhancerassociated lncRNAs, mediate the remodeling of chromatin landscape so as active enhancers to be gathered in close proximity to the target gene (e.g., HOTTIP) [21, 22].

Distinct mechanisms have been described regarding the function of lncRNAs in transcriptional regulatory programs. For example, these molecules are able to interact with a variety of transcription factors and to promote their activation/inactivation or to determine their subcellular localization. Moreover, lncRNAs can inhibit Pol II or conversely facilitate the binding of this enzyme to the gene promoter [23]. IncRNAs can also regulate gene expression in the posttranscriptional level. More precisely, lncRNAs act as miRNA "sponges" where, the lncRNA-miRNA base pairing modifies the latter's posttranscriptional effect and alters the expressional profile of their mRNA-target (e.g., HULC) [24]. Additionally, lncRNAs are implicated in alternative mRNA splicing. The most prominent example is the lncRNA MALAT1, which is proposed to interact with and control the activity of serine/arginine-rich protein, a central component of the splicing machinery [20].

lncRNAs have emerged as fine-tuners of cell differentiation and are implicated in various events in the life cycle of eukaryotes, ranging from gametogenesis to the formation of multicellular tissues and regulation of homeostasis. IncRNAs are also major parts of the transcriptional networks that induce or maintain ESC (Embryonic Stem Cells) pluripotency $[25,26]$. Moreover, lncRNAs are involved in embryogenesis, since they are essential for dosage compensation (e.g., XIST), genomic imprinting (e.g., Kcnqlotl), and the control of Hox gene expression [26]. The multifunctionality of lncRNAs allows them to take part in the complicated regulatory programs that govern the differentiation and development of distinct organs and tissues such as the central neural system, muscles, mammary gland, heart, skin, and adipose tissue $[21,25,26]$. 


\section{The Extensive Implication of IncRNAs in Cancer Pathobiology: Can It Be Translated to Clinical Practice?}

The established involvement of lncRNAs in a wide range of biological procedures has ushered in a new era of molecular genetics, where previously uncharacterized molecules seem to contribute to the etiology of human diseases. Modifications in the structure of lncRNAs, which are induced by large- or small-scale mutations, as well as their abnormal expression, have been linked with the onset of several pathological conditions and, most importantly, cancer [27, 28]. The aberrant expression of lncRNAs, across both solid and human hematological malignancies, brought these molecules to the forefront of cancer research and accelerated the investigation of their mechanistic roles in these multifactorial disorders. Dysregulated lncRNAs promote neoplastic transformation through the malfunction of common biological procedures such as epigenetic and transcriptional regulation, apoptosis, and epithelial-mesenchymal-transition (EMT). Although many functional aspects of lncRNAs still need to be clarified, accumulating in vitro and in vivo studies strengthen their role as oncogenes (e.g., HOTAIR, ANRIL, MALAT1, SRA, HULC, UCA1, PCA3, PCAT-1, PCGEM1, and PRNCR1) or tumor suppressors (LincRNA-p21, GAS5, MEG3, TERRA, PANDA, and TUG1) [29].

Several lncRNAs with oncogenic potential have already been reported. For instance, HOTAIR, promotes metastasis via a polycomb repression complex 2- (PRC2-) dependent pathway, simultaneously mediating the repression of several metastasis-suppressor genes and the activation of metastasisenhancing genes [30]. A recent study indicates that HOTAIR may serve as regulator of EMT [31] and, vice versa, its expression is controlled by signaling pathways and molecules that control EMT (WNT/ $\beta$-catenin signaling pathway and TGF-b) [29]. Similar to HOTAIR, ANRIL guides a PRCmediated silencing of the INK4b/ARF/INK4a locus. These genes encode for three tumor suppressor proteins (p15, p14, and p16) which play crucial roles in cell cycle block, senescence, and stress-induced apoptosis. Abnormal silencing of the aforementioned genes by ANRIL is a possible mechanism for cancer initiation. MALAT1 is also implicated in metastasis; chromosomal translocation is the main mechanism behind its marked upregulation in cancer [15]. Malfunction of MALAT1 affects cancer cells' mobility, invasiveness, and survival through the abnormal splicing of genes that are involved in oncogenesis- and metastasis-related procedures (e.g., WNT and MAPK signaling, cytoskeletal organization and cell cycle, EMT) $[29,32]$.

Contrariwise, tumor-suppressor lncRNAs have also been described. The currently known tumor suppressor lncRNAs are mainly implicated in apoptosis and cell cycle control, albeit they are characterized by dissimilar modes of action. For example, Linc-p21, PANDA, and TUG1 are involved in the p53 signaling and thus control the expression of genes that are related with apoptosis and cell cycle [33]. MEG3 also facilitates p53 signaling by activating and promoting p53 binding to the target genes. A p53-independent mode of action, which includes the control of $R b$ and the inhibition of cell proliferation, was also described recently for MEG3 [29]. GAS5 influences cell survival fate by activating the apoptotic machinery. Specifically, GAS5 competes with Glucocorticoid Response Elements (GRE) for binding to the glucocorticoid receptor (GR) and induces transcriptional suppression of GRrelated genes that act as inhibitors of apoptosis [34].

Apart from their regulatory role in oncogenic and tumorsuppressor pathways, the tissue- and cancer-specific expression patterns that lncRNAs commonly demonstrate [15] along with the advances in high-throughput expression analysis technologies have paved the way for the exploitation of these molecules in the clinical setting of oncology. Indeed, a bulk of experimental data denotes not only the dysregulation of lncRNAs in various cancers but also their association with patients' prognosis and response to treatment, rendering lncRNAs as an untapped source of diagnostic, prognostic, and predictive markers as well as a novel class of therapeutic targets.

The greatest example of the dynamics of lncRNAs as diagnostic biomarkers is the 2012-FDA approved PCA3 urine test for prostate cancer $[35,36]$. Interestingly, the lncRNA UCA1 (Urothelial Carcinoma Associated 1) can also be detected in urine sediment and represents a promising noninvasive diagnostic marker of bladder cancer [37]. Investigating the potential role of lncRNAs as prognostic and predictive markers has already provided encouraging results. In particular, HOTAIR is upregulated in both primary and metastatic breast tumors and is a robust marker of unfavorable prognosis regarding overall survival (OS) and metastasis [32]. MALAT1 is significantly overexpressed in metastasizing non-small cell lung cancer (NSCLC) tumors and serves as an independent prognostic indicator of poor survival for stage I NSCLC patients [38]. Moreover, H19 is a potent predictor of early recurrence in bladder cancer patients [24]. Expectations have also been raised regarding the potential of lncRNAs as predictive markers. For instance, XIST is correlated with the therapeutic response in ovarian cancer [15], and MALAT1 expression in osteosarcoma is correlated with poor response to chemotherapy [39].

The possible application of lncRNA-based therapies in clinical practice has attracted much pharmaceutical research interest and the first clinical trials are already underway (e.g., the DTA-H19 vector in bladder, ovarian, and pancreatic cancer) [40, 41]. Another approach, regarding the use of lncRNAs in cancer therapy, is the combined use of siRNAs against certain lncRNAs with conventional chemotherapeutic agents, which seems to result in effective sensitization of tumors to the latter [33, 42]. Antisense oligonucleotides represent an alternative option to achieve lncRNA targeting and recent studies demonstrated that this strategy can result in inhibition of MALAT1 and arrest of metastasis in mouse models [42].

\section{IncRNAs as a Novel Class of Cancer- Related Molecules and Novel Biomarkers in Digestive System Malignancies}

An exponentially increasing number of studies report that lncRNAs are deregulated in GI neoplasms. Analyses of 


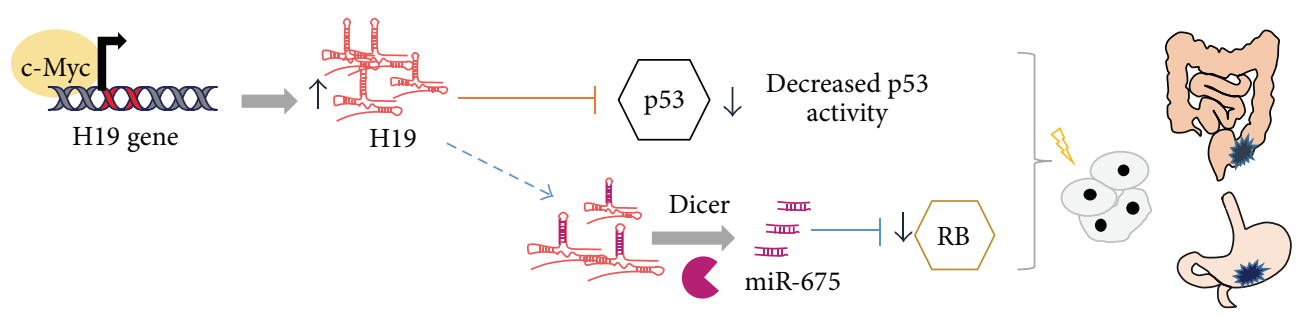

(a)
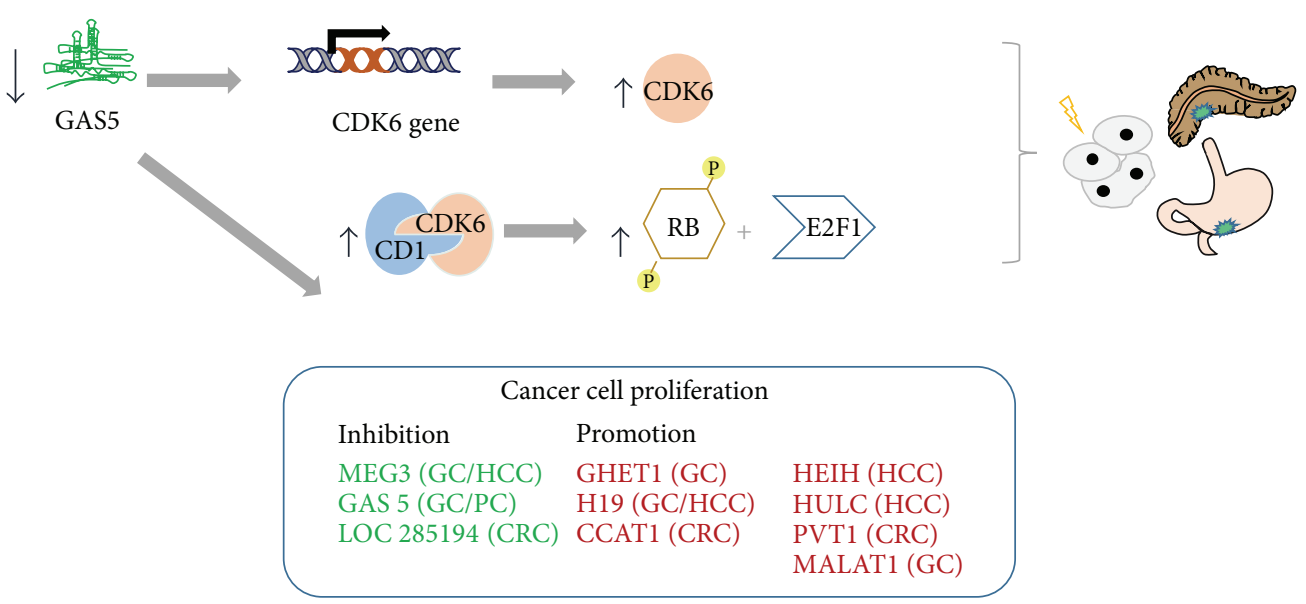

(b)

FIGURE 1: Representative mechanisms of lncRNAs implication in gastrointestinal cancer cell proliferation. (a) Promotion of gastric and colorectal cancer cell proliferation by H19. (b) Inhibition of gastric and pancreatic cell proliferation by GAS5; CRC: colorectal cancer, GC: gastric cancer, HCC: hepatocellular carcinoma, and PC: pancreatic cancer.

aberrantly expressed lncRNAs, though mechanistic studies, provide new insights into their ubiquitous implication in pathways that govern hallmark processes of cancer, including cell proliferation, evasion of apoptosis, tumor invasion, and metastasis.

5.1. Mechanistic Aspects of IncRNA Involvement in Digestive System Malignancies. IncRNAs play important roles at initial stages of GI tract tumorigenesis by either promoting or repressing cancer cell proliferation (Figure 1). The lncRNA, GHET1, is involved in cell proliferation in GC. Yang et al. demonstrated that enhanced expression of GHET1 promotes cell proliferation both in vitro and in vivo, whereas the inhibition of GHET1 expression via siRNA, hinders cell proliferation in vitro. In a series of experiments, it was shown that GHET1 exerts its proproliferative role through upregulation of c-Myc expression, by binding IGF2BP1 and enhancing the interaction between IGF2BP1 protein and c-Myc mRNA [43]. H19 is another lncRNA, which affects cancer cell proliferation. The $\mathrm{H} 19$ gene is paternally imprinted and is expressed by the maternal allele. Loss of H19 imprinting [44] and overexpression was reported in several GI cancers such as GC, hepatocellular (HCC), and esophageal carcinomas [45]. Several mechanisms have been proposed for the oncogenic activity of H19 in GI cancers. In GC cell lines, ectopic expression of $\mathrm{H} 19$ results in increased cell proliferation and depletion of its expression promotes cell apoptosis. The mechanism underlying these observations was found to be the association of H19 with p53, which in turn affects the tumor suppressive function of the latter [46]. Additionally, a different study revealed that $\mathrm{H} 19$ expression can be induced by the oncogene c-Myc in vitro, and in GC tissue samples the expression of H19 positively correlates with c-Myc levels. The same research group revealed that depletion of $\mathrm{H} 19$ results in decreased proliferation of GC cells [47]. Furthermore, H19 is the primary precursor of miR-675, which in turn acts as the mediator of the H19 tumorigenic function in CRC cells via targeting the tumor suppressor protein RB [45]. Similarly to H19, c-Myc can also induce the transcription of CCAT1, also known as CARLo-5 (cancer-associated region long noncoding RNA), transcription and its ectopic expression was able to promote CRC cell proliferation in vitro [48]. CARLo-5 expression is regulated by a physical interaction between the $M Y C$ enhancer region and the active regulatory region of the CARLo-5 promoter. By performing siRNA-mediated silencing of CARLo-5 in various CRC cell lines, Kim et al. suggested that CARLo-5 inhibits G1 phase arrest by regulating the expression of several cell-cycle-related genes such as CDKN1A [49]. A similar study in GC cells, confirmed the regulatory role of CARLo-5 in cell proliferation and apoptosis. Briefly, it was found that knockdown of CARLo-5 resulted in decreased expression of PCNA (proliferating cell nuclear antigen), which is essential for DNA replication and increased synthesis of the cellcycle regulators p16, p21, and p27. Moreover, siCARLo-5 cells were characterized by common features of late apoptosis and altered expression profiles of apoptosis-related proteins [50]. 
Additionally, the lncRNA HEIH plays an important role in cell-cycle regulation. In HCC cells, depletion of HEIH resulted in reduced cell proliferation and contributed to cellcycle arrest mainly by p16, $\mathrm{p} 27$, and $\mathrm{p} 21$ protein upregulation. It was postulated that the association of $\mathrm{HEIH}$ with the enhancer of zeste homolog 2 (EZH2) is required for the repression of the EZH2 target genes, which include cellcycle regulation genes [51]. HULC is a strongly upregulated mRNA-like lncRNA in liver cancer that localizes to the cytoplasm and is associated with ribosomes. Interestingly, knockdown of HULC RNA in HCC cells results in a significant up- or downregulation of different genes that have been previously reported to be involved in liver carcinogenesis [52]. HULC can also repress the expression of the tumor suppressor p18, enhancing in this way HCC proliferation mediated by Hepatitis B virus X protein ( $\mathrm{HBx}$ ) [53]. The transcription factor CREB can activate HULC expression in a cancer-specific manner through a regulatory loop which involves miR-372 and its target gene cAMP-dependent protein kinase catalytic subunit b (PRKACB) [54]. Additionally, knockdown of PVT1 inhibits cell proliferation in vitro and promotes apoptosis through the activation of TGF- $\beta$ signalling pathway-related genes such as SMAD4 [55]. Wang et al., have shown that RNA interference-mediated silencing of MALAT1 in GC cells, results in cell cycle arrest in G0/G1 phase and suppression of cell proliferation. MALAT1 was found to affect GC cell proliferation partly through the modulation of expression levels and nuclear distribution of SF2/ASF, a member of the serine/arginine (SR) splicing factors. This observation, in addition to the fact that MALAT1 is localized in nuclear speckles, implies that it may affect the alternative splicing of pre-mRNAs, through the modulation of SR factors [56].

On the contrary, overexpression of GAS5 can reduce GC cell proliferation and induce apoptosis both in vitro and in vivo, whereas knockdown of GAS5 can promote cell proliferation. Interestingly, inhibition of GAS5 expression resulted in increased protein levels of E2F1 and Cyclin D1, which are two major players in the retinoblastoma protein $(\mathrm{pRB})$ pathway, as well as in decreased P21 levels which has a critical role in cell cycle arrest [57]. In pancreatic cancer cells, inhibition of GAS5 expression increases CDK6 protein levels thereby facilitating cell cycle progression [58]. MEG3 is abundantly expressed in normal human tissues and its expression is often lost in cancer, suggesting that it functions as a tumor suppressor lncRNA. Transfection of GC and CRC cells with MEG3 resulted in inhibition of cell proliferation and promotion of apoptosis, partly through activation of p53 $[59,60]$. Ectopic expression of MEG3 in nude mice confirmed the tumor suppressive nature of this IncRNA, since MEG3 upregulation resulted in inhibition of CRC proliferation [60]. Further in vitro studies indicate that overexpression of MEG3 activates p53 and is able to inhibit cell proliferation, through the stimulation of endogenous p53 target genes [61]. In a series of in vitro experiments, Braconi et al. demonstrated that HCC cells with enforced MEG3 expression, have reduced capacity for anchorage-dependent and -independent growth; MEG3 expression induced also apoptosis in these cell lines. The same study showed that hypermethylation of MEG3 promoter is the main mechanism behind the decreased expression of
MEG3 in HCC [62]. In GC, it was shown that miR-148a can indirectly induce the overexpression of MEG3, through downregulation of DNA methyltransferase 1, thereby inhibiting GC cell proliferation [63]. Moreover, loc285194 which is a p53-regulated tumor suppressor IncRNA was found to inhibit CRC cell growth both in vitro and in vivo [64].

IncRNAs are key players in the complex and multistep process of cancer progression, invasion, and metastasis (Figure 2). HOTAIR epigenetically regulates the expression of essential metastasis-suppressor genes through the coordination of histone modification complexes, PRC2 and LSD1. In CRC, HOTAIR closely correlates with members of the PRC2 complex and ectopic overexpression of HOTAIR promoted invasion of cancer cells in vitro [65]. Additionally, HOTAIR may promote migration and invasion of HCC cells, partially through the negative regulation of RNA binding motif protein 38 (RBM38) [66]. A different mechanism for HOTAIR oncogenic activity was provided by a recent study using in vitro assays in HCC. Silencing of HOTAIR expression reduced HCC cell proliferation and negatively regulated the expression levels of matrix metalloproteinase-9 (MMP9) and angiogenic factor VEGF, which are known to be involved in metastasis and angiogenesis, respectively [67]. Likewise, in GC cell lines, knock-down of HOTAIR, reduced invasiveness and the expression of MMP1 and MMP3. Another interesting finding was that HOTAIR silencing reversed EMT through regulation of Snail, which is one of the main transcription factors that controls EMT and cell motility [31]. HOTAIR can also act as an endogenous miRNA "sponge" which restrains miR-331-3p activity on HER2, thus enhancing HER2 oncogenic activity in GC cells [68]. Ma et al. showed that in gallbladder cancer cells HOTAIR is a direct target of c-Myc and its activity may be partially explained by the downregulation of miR-130a, via promoter methylation [69]. MALAT1 is one of the most prominent oncogenic lncRNAs and is reported to be highly expressed in several GI cancers such as GC, CRC, and HCC. A number of studies provided evidence that ectopic expression of MALAT1 promotes proliferation and migration of cell lines in vitro and enhances tumor growth and metastasis in vivo. In CRC cells, MALAT1 was found to bind the tumor suppressor SFPQ protein, which in turn leads to release of the oncogenic PTBP2 from the SFPQ/PTBP2 complex, thereby promoting cell proliferation and migration. Additionally, the same research group showed that MALAT1 could promote metastasis of CRC cells in nude mice [70]. The abovementioned effects of MALT1 were confirmed by a recent study where the newly identified mechanism for gene induction, named RNAa, was used. According to these results, MALAT1 upregulation induces CRC cell growth and cell cycle G1/S phase transition in vitro and promotes CRC growth in vivo. Additionally, using orthotopic tumor models in nude mice, it was found that MALAT1 induction resulted in a significant increase of tumor metastatic potential. These effects were attributed to the induction of MALAT1 target gene AKAP-9 [71]. The overexpression of CCAT2 promotes tumor growth and metastasis in CRC mouse xenograft models. It was demonstrated that CCAT2 directly interacts with the transcription factor TCF7L2, thereby activating WNT signaling pathway, while CCAT2 is also a downstream target 


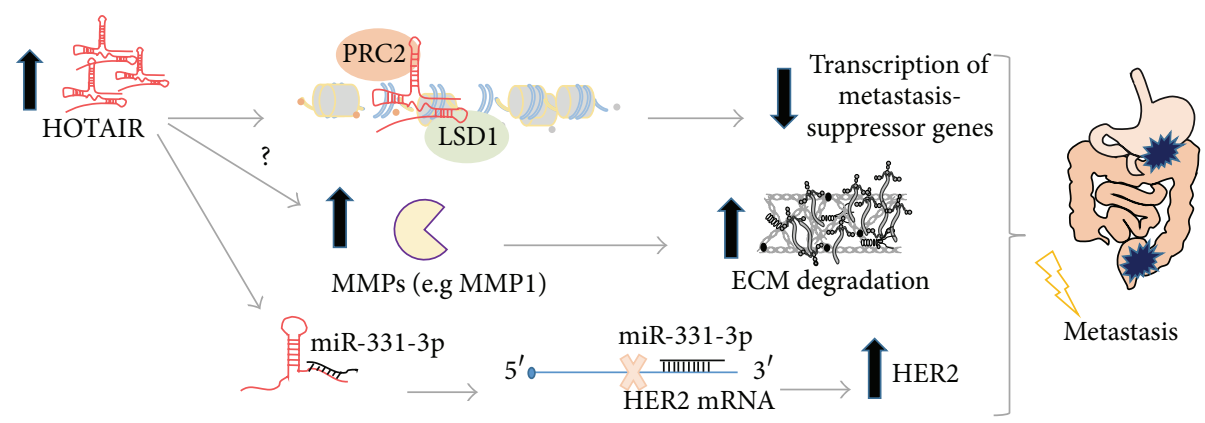

(a)

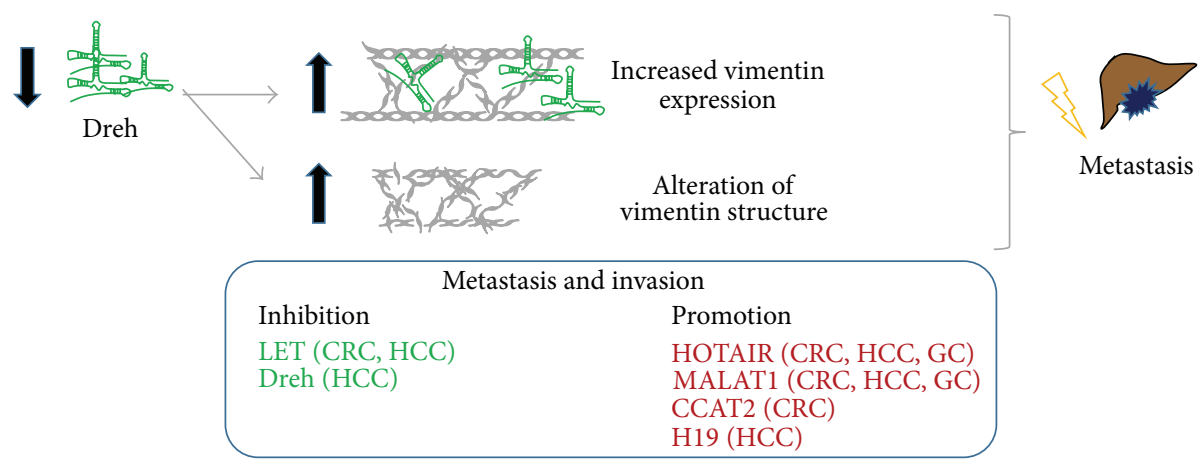

(b)

FIGURE 2: Representative mechanisms of lncRNAs implication in gastrointestinal cancer invasion and metastasis. (a) Promotion of gastric and colorectal cancer invasion and metastasis by HOTAIR. (b) Inhibition of hepatocellular carcinoma cell invasion by Dreh; CRC: colorectal cancer, GC: gastric cancer, HCC: hepatocellular carcinoma, and PC: pancreatic cancer.

of WNT. Additionally, CCAT2 can increase the expression of WNT target genes including MYC and MYC-regulated miRNAs such as miR-17-5p, and miR-20a, by TCF7L2-mediated transcriptional regulation [72]. Moreover, H19 is highly expressed in liver cancer cell lines, and hypoxic conditions strongly upregulate $\mathrm{H} 19$ levels. Tumor hypoxia affects cancer progression and metastasis, and it renders tumors resistant to anticancer therapy [73]. In contrast with the above, Zhang et al. demonstrated that H19 may activate miR-200 family members, resulting in subsequent suppression of their target genes, E-cadherin transcriptional repressors (ZEB1/2). The miR-200 family plays an important role in repression of EMT, through direct targeting of ZEB1/2 [74].

On the other side of the spectrum, LET acts as an inhibitor of metastasis in HCC under conditions of hypoxia. This lncRNA is downregulated in certain GI cancers, including HCC and CRC and its mechanism of action has been studied in orthotopic tumor models in nude mice. According to these results LET is a component of a positive feedback loop which includes the hypoxia-inducible factor 1, alpha subunit (HIF-1a) the histone deacetylase 3 (HDAC3), and the NF90. Upon hypoxia, LET is suppressed by HDAC3, and LET-NF90 interaction is disturbed. As a consequence, the stabilization of NF90 is enhanced, resulting in increased expression of HIF-1a, which has a well-documented role in hypoxia-induced invasion and metastasis [75]. The depletion of Dreh expression is found to be associated with increased invasion in vitro and in vivo. A mechanistic study showed that Dreh exerts its action through the binding of vimentin, a major cytoskeletal protein, thus leading to arrest of metastasis. In Dreh-transfected cells, repression of vimentin expression and changes regarding the vimentin organization and localization have been reported, which are related with inhibition of cell migration [76]. LEIGC (lower expression in gastric cancer) is a newly identified lncRNA with a tumor suppressive role in GC, which prevents cell migration and EMT in vitro. In more details, Han et al. performed gain- and loss-of-function experiments in GC cell lines and found that LEIGC silencing resulted in increased migration potential of the cells and was accompanied by morphological characteristics which are indicative of transdifferentiation from epithelial to mesenchymal phenotype. The latter was confirmed by both decreased expression of epithelial cell-related genes and proteins, such as $C D H 1$ and E-cadherin, and upregulation of mesenchymal-cell markers like snail, slug, twist and zeb, and vimentin [77].

Given the active involvement of IncRNAs in cancer progression, along with their aberrant expression which is often associated with clinicopathological characteristics and prognosis of cancer patients and their tissue- or cancerspecific expression patterns, it is probable that lncRNAs could soon outperform the currently suggested mRNA or protein markers. lncRNAs have just begun to prove their dynamics as diagnostic, prognostic, and predictive molecular 
TABLE 1: An overview of the biomarker potential of lncRNAs for colorectal and gastric cancer.

\begin{tabular}{|c|c|c|c|c|c|}
\hline $\operatorname{lncRNA}$ & Cancer type & Sample & Expression & Clinical application & Reference \\
\hline $\begin{array}{l}\text { CRNDE (splice } \\
\text { variants) }\end{array}$ & CRC & Tissue & $\mathrm{Up}$ & Diagnosis & {$[78]$} \\
\hline CRNDE h & CRC & Plasma & Up & Diagnosis & {$[78]$} \\
\hline ncRuPAR & $\mathrm{CRC}$ & Tissue & Down & Diagnosis & {$[79]$} \\
\hline MALAT1 & CRC & Tissue & $\mathrm{Up}$ & Unfavorable prognosis & {$[87]$} \\
\hline PVT1 & CRC & Tissue & Up & Unfavorable prognosis & {$[55]$} \\
\hline UCA1 & CRC & Tissue & $\mathrm{Up}$ & Unfavorable prognosis & {$[88]$} \\
\hline PCAT-1 & $\mathrm{CRC}$ & Tissue & Up & Unfavorable prognosis & [89] \\
\hline $91 \mathrm{H}$ & CRC & Tissue & $\mathrm{Up}$ & Unfavorable prognosis & {$[90]$} \\
\hline LOC285194 & CRC & Tissue & Down & Favorable prognosis & {$[93]$} \\
\hline ncRAN & CRC & Tissue & Down & Favorable prognosis & {$[94]$} \\
\hline RP11-462C24.1 & $\mathrm{CRC}$ & Tissue & Down & Favorable prognosis & [95] \\
\hline u.73 & CRC & Tissue & Down & Favorable prognosis & {$[96]$} \\
\hline \multirow{2}{*}{ H19 } & GC & Plasma & $\mathrm{Up}$ & Diagnosis & {$[114]$} \\
\hline & GC & Tissue & Up & Unfavorable prognosis & {$[104]$} \\
\hline Linc00152 & GC & Gastric juice & Up & Diagnosis & {$[115]$} \\
\hline AA174084 & GC & Gastric juice & Up & Diagnosis & {$[110]$} \\
\hline $\mathrm{AC} 138128.1$ & GC & Tissue & Down & Diagnosis & {$[109]$} \\
\hline ANRIL & GC & Tissue & Up & Unfavorable prognosis & {$[105]$} \\
\hline GHET1 & GC & Tissue & Up & Unfavorable prognosis & {$[43]$} \\
\hline HULC & GC & Tissue & Up & Unfavorable prognosis & {$[106]$} \\
\hline CCAT2 & GC & Tissue & Up & Unfavorable prognosis & {$[120]$} \\
\hline FENDRR & GC & Tissue & Down & Favorable prognosis & {$[111]$} \\
\hline GACAT1 & GC & Tissue & Down & Diagnosis/favorable prognosis & {$[112]$} \\
\hline LET & GC & Tissue & Down & Favorable prognosis & {$[121]$} \\
\hline BM742401 & GC & Tissue & Down & Favorable prognosis & {$[122]$} \\
\hline \multirow{2}{*}{$\begin{array}{l}\text { CCAT1 } \\
\text { (CARLo-5) }\end{array}$} & CRC & Tissue/blood & $\mathrm{Up}$ & $\begin{array}{c}\text { Screening/diagnosis/monitoring } \\
\text { of disease progression }\end{array}$ & {$[81]$} \\
\hline & GC & Tissue & Up & Unfavorable prognosis & {$[119]$} \\
\hline \multirow{2}{*}{ HOTAIR } & CRC & Tissue/blood & Up (blood) & Unfavorable prognosis & {$[65,85,86]$} \\
\hline & GC & Tissue & Up & Unfavorable prognosis & {$[100,101,103]$} \\
\hline \multirow{2}{*}{ LSINCT5 } & CRC & Tissue & Up & Unfavorable prognosis & {$[92]$} \\
\hline & GC & Tissue & Up & Unfavorable prognosis & [92] \\
\hline \multirow{2}{*}{ GAS5 } & CRC & Tissue & Down & Favorable prognosis & {$[97]$} \\
\hline & GC & Tissue & Down & Favorable prognosis & {$[57]$} \\
\hline \multirow{2}{*}{ MEG3 } & CRC & Tissue & Down & Favorable prognosis & {$[60]$} \\
\hline & GC & Tissue & Down & Favorable prognosis & {$[59]$} \\
\hline
\end{tabular}

markers for GI malignancies and further elucidation of specific mechanisms of action can pave the way for designing novel anticancer therapeutic approaches.

\subsection{IncRNAs as Biomarkers for Colorectal Cancer}

5.2.1. IncRNAs in CRC Screening and Diagnosis. A number of lncRNAs may serve as molecular markers for CRC diagnosis (Table 1). Graham et al. showed that CRNDE splice variants are upregulated in early stage neoplastic colorectal tissues including adenomas and adenocarcinomas. Additionally, CRNDE splice variant h expression levels could efficiently discriminate adenomas from normal tissues and measurement of CRNDE-h RNA levels in plasma samples using qPCR resulted in a sensitivity of $87 \%$ and specificity of $93 \%$, for detecting CRC [78]. The diagnostic performance of a newly detected lncRNA ncRuPAR was recently studied by Yan et al. The expression of ncRuPAR is downregulated in cancer and can effectively differentiate CRC from benign tissues, with high sensitivity [79]. 
CCAT1 has also been proposed as a potential biomarker for screening and diagnosis of CRC. This lncRNA is found to be overexpressed in tissues and in peripheral blood samples obtained from CRC patients compared to healthy controls [80]. Moreover, CCAT1 expression was studied across a spectrum of tissues from different stages of CRC progression. CCAT1 upregulation is evident in premalignant conditions and in all disease stages including distant CRC metastasis, suggesting that CCAT1 can also be used for monitoring of disease progression [81]. Interestingly, Kam et al. developed a CCAT1-specific peptide nucleic acid (PNA) based molecular beacon and showed that it can serve as a valuable tool for both imaging and in situ detection of CRC [82]. Single nucleotide polymorphisms (SNP) in lncRNA genes are associated with CRC susceptibility. In particular, two SNPs located in the lncRNA PRNCR1 gene (rs13252298 and rs1456315) are connected with decreased risk for CRC, while patients harboring two different SNPs, namely, rs7007694C and rs16901946G, have low risk to develop poorly differentiated CRC. On the contrary, the rs1456315G SNP is linked to increased risk for the development of CRC with poor differentiated status [83]. Similarly, Xue et al. reported that a HOTAIR SNP is associated with risk of CRC development. Briefly, the rs7958904 CC genotype is related with decreased risk of CRC compared to the rs7958904 GG genotype [84].

5.2.2. IncRNAs in CRC Prognosis. A recent study demonstrated that increased HOTAIR expression, as assessed by qPCR in CRC tissues, positively correlates with advanced tumor stage, high recurrence rate, and short metastasisfree and OS intervals of the patients $[65,85]$. Additionally, HOTAIR overexpression in blood samples obtained from CRC patients is significantly associated with reduced OS [86], a fact that strengthens HOTAIRs' vast potential as a prognostic biomarker. The prognostic significance of MALAT1 in stage II/III CRC patients is also revealed. Patients with elevated MALAT1 expression have significantly higher risk for metastasis after radical surgery, and MALAT1 expression is an independent prognostic factor of both disease-free survival (DFS) and OS of CRC patients [87]. Furthermore, PVT1 expression is found to be a powerful prognostic indicator in CRC. Patients with high PVT1 expression levels present a more adverse outcome as indicated by shorter OS periods, compared to those with low PVT1 expression. Moreover, multivariate analysis revealed that PVT1 expression in CRC predicts an increased risk of death, independently of important clinicopathological factors [55]. UCA1, exhibits high expression in CRC tissues and correlates with larger tumor size and unfavorable prognosis of the CRC patients [88]. Similarly, overexpression of PCAT-1 is an indicator of distant metastasis and an independent predictor of poor OS of patients [89]. The levels of lncRNA 91H expression are significantly upregulated in CRC compared to noncancerous tissues, and $91 \mathrm{H}$ constitutes an independent predictor of poor prognosis in CRC [90]. The expression of BANCR is positively associated with lymph node metastasis and tumor stage; however, its prognostic significance, in terms of patients' survival, has not yet been reported [91]. LSINCT5 upregulation is associated with more aggressive disease phenotypes as well as with shorter DFS and DSS periods, revealing its possible role as an indicator of poor prognosis in CRC [92].

On the other hand, the expression levels of numerous lncRNAs are reported to be downregulated in CRC. Reduced expression levels of LOC285194 are associated with more aggressive features of tumors and correlate significantly with shorter DFS [93]. Low ncRAN levels are detected in high histological grade tumors and more importantly decreased expression of this lncRNA seems to be an independent prognostic factor of poor OS [94]. Focusing on RP11-462C24.1, low expression levels are found in patients with metastasis and multivariate analysis revealed that decreased RP11-462C24.1 expression can serve as an independent prognosticator of poor DSS of CRC patients [95]. The expression of a novel class of lncRNAs, transcribed ultraconserved regions ( $\mathrm{T}$ UCRs), namely, uc.73 and uc.388, is reported to decrease in CRC and uc.73 is found to be associated with OS of CRC patients [96]. Additionally, patients with low GAS5 expression had significantly shorter OS than those with high GAS5 expression and GAS5 expression was identified as an independent indicator of CRC prognosis [97]. Likewise, MEG3 downregulation is associated with advanced TNM stages, deeper tumor invasion and inferior OS intervals. Moreover, MEG3 is an independent indicator of favorable prognosis in terms of OS in CRC patients [60].

Recently, a prognostic lncRNA signature consisting of six different lncRNAs was identified by $\mathrm{Hu}$ et al., using a microarray data mining approach and CRC data sets from GEO database. Based on this signature they divided CRC patients into high- and low-risk groups and showed that patients belonging to the former group had shorter DFS [98].

\subsection{IncRNAs as Biomarkers for Gastric Cancer}

5.3.1. IncRNAs in GC Diagnosis. Many studies imply the presence of differential levels of several lncRNAs between cancerous and noncancerous conditions, not only in tissues but in biofluids as well $[46,57,59,68,99-113]$ (Table 1). Arita et al. showed not only a significant overexpression of H19 in the plasma of GC patients compared to healthy controls, but also a substantial reduction of the levels of circulating H19 in plasma after gastrectomy [114]. The expression levels of Linc00152 were analyzed in gastric juice from GC patients and normal controls and a significant increase of Linc00152 in patients was observed [115]. AA174084 is another lncRNA with possible diagnostic value in GC, as it was significantly upregulated in gastric juice from GC patients compared to the gastric juice derived from patients with other gastric disorders or to normal mucosa [110]. According to the analysis of global expression profile of lncRNAs in GC, many lncRNAs exhibited different expression patterns in paired GC and normal adjacent tissue samples [116, 117]. Recently, Mizrahi et al. compared the expression levels of CCAT1 in GC tissues to normal ones, obtained from patients with morbid obesity, revealing a statistical significant increase in GC patients [118].

5.3.2. IncRNAs in GC Prognosis. HOTAIR, seems to be an indicator of poor prognosis in GC patients, since its overexpression was associated with advanced TNM and pathological stage, large tumor size, increased tumor invasiveness, 
and metastatic potential as well as with an adverse outcome in terms of OS [68, 99-103]. Moreover, increased expression of H19 was significantly associated with shorter OS periods; highlighting its value as a biomarker of unfavorable prognosis [104]. A recent study revealed that ANRIL upregulation is an independent marker of unfavorable prognosis in GC, since its expression positively correlates with advanced disease stage and large tumor size as well as with limited DFS and OS [105]. CCAT1 and CCAT2 are also considered as indicators of poor prognosis. Specifically, high expression levels of CCAT1 were associated with tumor growth, lymph node metastasis [119], and advanced TNM stage [50], whereas CCAT2 overexpression was related with increased metastatic and invasive potential of the tumor. Moreover CCAT2 is an independent prognostic indicator of decreased DFS and OS intervals in GC patients [120]. Similarly, HULC upregulation was associated with increased tumor metastatic potential, unraveling the role of this lncRNA as an indicator of poor prognosis [106]. LSINCT5 is also an independent marker of unfavorable prognosis and its expression positively correlates with several clinicopathological parameters of poor prognosis in GC (advanced TNM stage, large tumor size, lymph node metastasis, and deeper invasion depth) as well as with shorter DFS and DSS periods [92]. Finally, overexpression of GHET1 is significantly related to tumor size and invasion and to poor OS of the patients [43].

On the other hand, lncRNAs such as FENDRR, MEG3, LET, GAS5, BM742401, and GACAT1 were found to represent indicators of favorable prognosis. In particular, FENDRR decreased expression seemed to be an independent prognostic indicator of decreased DFS and OS intervals and was associated with deeper tumor invasion, advanced tumor stage, and lymph node metastasis [111]. Concerning MEG3, its downregulation was associated not only with markers of poor prognosis, namely, large tumor size, advanced pathological stage and increased depth of invasion, but also with shorter OS [59]. Similarly, LET expression was negatively associated with tumor invasiveness and TNM stage, and decreased LET expression levels consist an independent prognostic marker of limited OS of GC patients [121]. GAS5 and BM742401 are additional markers of favorable GC patients' prognosis since decreased expression levels were associated with poorer survival $[57,122]$. In advanced disease stages, the downregulation of GACAT1 was related with increased tumor invasiveness and metastatic potential [112] (Table 1).

\section{4. $\ln \mathrm{R} R$ As as Biomarkers for \\ Hepatocellular Carcinoma (HCC)}

5.4.1. IncRNAs in HCC Diagnosis. Xie et al. reported that HULC plasma levels were significantly higher in HCC patients than in the healthy control group and that HULC tissue expression demonstrated adequate sensitivity and specificity to discriminate malignant from normal liver tissues [123]. Additionally, HULC seems to be a hepatocarcinogenesis-specific lncRNA since it is only slightly overexpressed in cirrhotic liver disorders. Recently, it was reported that lncRNA-uc003wbd and lncRNA-AF085935 serum levels can effectively discriminate $\mathrm{HCC}$ from $\mathrm{HBV}$ infection as well as HCC and HBV-affected patients from healthy volunteers [124]. IncRNAs such as HULC, HOTAIR, H19, HEIH, MVIH, and PVT1 are upregulated in HCC, in contrast to MEG3, hDREH, GAS5, and LET that exhibit lower expression in this malignancy [125]. Particularly, HEIE seems to be linked with pathological conditions of liver, since it was found to be overexpressed both in cancer and cirrhosis [51]. According to a recent microarray-based analysis of the expression profile of lncRNAs in HCC, 214 lncRNAs exhibited aberrant expression in HCC tissues compared to normal ones [126].

5.4.2. IncRNAs in HCC Prognosis. HULC is a marker of poor prognosis in HCC since patients with higher Edmondson grades or with positive HBV (Hepatitis B Virus) status demonstrated higher HULC levels both in plasma and tissue [123]. HOTAIR upregulation was associated with lymph node metastasis [67] and large tumor size [127] as well as inferior DFS after resection or transplantation [125], revealing its potential as marker of unfavorable patients' prognosis. HEIH is an independent prognostic indicator of shorter OS and DFS in HBV-related HCC [51], whereas MVIH is an independent marker of poor outcome after hepatectomy, as its upregulation is related to lower DFS and OS probabilities [128]. According to recent data, four additional lncRNAs, namely, URCH, lncRNA-ATB, HOTTIP, and PVT1, seem to have a role as markers of poor prognosis. Specifically, increased URCH levels were associated with inferior OS intervals [129]; upregulation of lncRNA-ATB exhibited a strong association with liver cirrhosis and microvascular and macrovascular invasion and encapsulation [130] and HOTTIP levels, along with the mRNA HOXA13, were associated with patients' clinical progression and were able to predict disease outcome [131]. Regarding PVT1, its expression levels were analyzed in tissues obtained from patients subjected to liver transplantation, and significant associations of PVT1 overexpression with advanced TNM stages, higher recurrence rate, and increased AFP levels were observed. Additionally, PVT1 seems to be an independent predictor of poor DFS in HCC patients after liver transplant [132].

As opposed to the lncRNAs discussed above, hDREH, LET, and GAS5 may hold value as biomarkers of favorable prognosis for HCC. In particular, hDREH downregulation is associated with shorter DFS and OS intervals of HCC patients [76]. Moreover, LET decreased expression was associated with tumor micrometastasis and encapsulation [125], whereas GAS5 downregulation was associated with markers of poor prognosis (tumor size, lymph node metastasis, and clinical stage) and emerged as an independent predictor of 5-year DFS [133] (Table 2).

5.5. IncRNAs as Prognostic Biomarkers for Pancreatic Cancer. The clinical relevance of lncRNA MALAT1, in pancreatic ductal adenocarcinoma (PDAC), was investigated by Liu et al. The expression of MALAT1 was measured in FFPE tissues using qPCR and was found to be significantly higher in PDAC compared to adjacent noncancerous tissues. Additionally, MALAT1 expression levels were positively correlated with tumor size, stage, and depth of invasion and the overexpression of MALAT1 was independently associated with 
TABLE 2: An overview of the biomarker potential of lncRNAs for hepatocellular and pancreatic cancer.

\begin{tabular}{|c|c|c|c|c|c|}
\hline $\operatorname{lncRNA}$ & Cancer type & Sample & Expression & Clinical application & Reference \\
\hline HULC & HCC & Tissue/plasma & Up & $\begin{array}{c}\text { Diagnosis/unfavorable } \\
\text { prognosis }\end{array}$ & {$[123]$} \\
\hline uc003wbd & HCC & Serum & Up & Diagnosis & {$[124]$} \\
\hline AF085935 & HCC & Serum & $\mathrm{Up}$ & Diagnosis & {$[124]$} \\
\hline HEIE & $\mathrm{HCC}$ & Tissue & $\mathrm{Up}$ & Unfavorable prognosis & {$[51]$} \\
\hline MVIH & HCC & Tissue & $\mathrm{Up}$ & Unfavorable prognosis & {$[128]$} \\
\hline $\mathrm{URCH}$ & HCC & Tissue & Up & Unfavorable prognosis & [129] \\
\hline lncRNA-ATB & $\mathrm{HCC}$ & Tissue & Up & Unfavorable prognosis & {$[130]$} \\
\hline HOTTIP & HCC & Tissue & $\mathrm{Up}$ & Unfavorable prognosis & {$[131]$} \\
\hline hDREH & $\mathrm{HCC}$ & Tissue & Down & Favorable prognosis & {$[76]$} \\
\hline LET & $\mathrm{HCC}$ & Tissue & Down & Favorable prognosis & {$[125]$} \\
\hline GAS5 & $\mathrm{HCC}$ & Tissue & Down & Favorable prognosis & {$[133]$} \\
\hline MALAT1 & PDAC & FFPE tissues & Up & Unfavorable prognosis & {$[134,135]$} \\
\hline ENST00000480739 & PDAC & Tissue & Down & Favorable prognosis & {$[3]$} \\
\hline BC008363 & PDAC & Tissue & Down & Favorable prognosis & {$[138]$} \\
\hline \multirow{2}{*}{ HOTAIR } & HCC & Tissue & Up & Unfavorable prognosis & {$[127]$} \\
\hline & Pancreatic cancer & Tissue & Up & Unfavorable prognosis & [136] \\
\hline \multirow{2}{*}{ PVT1 } & HCC & Tissue & Up & Unfavorable prognosis & {$[132]$} \\
\hline & PDAC & Tissue & Up & Unfavorable prognosis & {$[137]$} \\
\hline
\end{tabular}

poorer disease-specific survival of PDAC patients [134]. In concordance with the aforementioned results, Pang et al. reported that MALAT1 overexpression in pancreatic cancer tissues is related to aggressive phenotypes of the disease and is an independent marker of poor prognosis regarding OS [135]. HOTAIR is also highly expressed in aggressive pancreatic tumors and the respective patients had significantly shorter OS [136]. Furthermore, lncRNA PVT1 is an independent marker of decreased OS in PDAC and it is associated with clinical stage and N-classification [137].

A novel lncRNA ENST00000480739 was recently identified through lncRNA expression microarray. The expression of this lncRNA is significantly lower in PDAC patients with lymph node metastasis and its expression is associated with prolonged OS [3]. Likewise, the lncRNA BC008363 is found to be downregulated in PDAC compared to paired noncancerous tissues. Patients with high expression levels had significantly better OS, suggesting that BC008363 is a biomarker of favorable prognosis in PDAC [138]. Several lncRNAs are implicated in metastasis in pancreatic cancer, suggesting that they are associated with unfavorable prognosis of patients. For instance, Tahira et al. identified a set of 134 lncRNAs that correlate with metastasis in PDAC [139]. A different study revealed that $\mathrm{H} 19$ is overexpressed in PDAC tissues and in primary pancreatic tumors that subsequently metastasize [140] (Table 2).

\subsection{IncRNAs as Biomarkers for Esophageal Cancer}

5.6.1. IncRNAs in Esophageal Cancer Screening and Diagnosis. Esophageal cancer (EC) occurs in two main histological subtypes, esophageal squamous cell carcinoma (ESCC) and esophageal adenocarcinoma (EAC), which differ in incidence and etiology. Several lncRNAs are reported to be aberrantly expressed in esophageal cancer. For instance, the expression of ANRIL [141] and POU3F3 [142] is found to be higher in ESCC compared to noncancerous tissues. Interestingly, Tong et al. reported a significant increase of POU3F3 plasma levels in ESCC patients compared to healthy controls. They also found that the combination of POU3F3 with the classic tumor marker, SCCA, can aid in early detection of ESCC, revealing its role as a promising diagnostic tool for population screening [143]. Furthermore, a functional genetic polymorphism in lincRNA-uc003opf.1 exon was found to be associated with susceptibility to ESCC. In particular, patients with rs11752942AG and GG genotypes have lower risk for ESCC compared to rs11752942AA, revealing their value as markers for screening high-risk populations [144]. In EAC, HNF1AAS1 is strongly upregulated in cancerous tissues compared to corresponding normal ones, revealing its possible diagnostic value for this subtype of esophageal cancer [145]. The expression of AFAP1-AS1 is markedly increased in EAC compared to normal esophageal tissues and hypomethylation is responsible for the observed upregulation [146]. Recently, Fassan et al., using microarray analysis, identified T-UCR signatures that were deregulated across the squamous epithelia to Barrett's adenocarcinoma sequence of neoplastic progression, suggesting that these signatures may serve as tools for assessing risk of developing Barrett's-esophagus associated cancer [147].

5.6.2. IncRNAs in Esophageal Cancer Prognosis and Treatment Response Prediction. Similarly to other GI cancers, an important correlation with limited DFS and OS of ESCC patients has been observed for HOTAIR expression [148]. 
TABLE 3: An overview of the biomarker potential of lncRNAs for esophageal cancer.

\begin{tabular}{|c|c|c|c|c|c|}
\hline $\operatorname{lncRNA}$ & Cancer type & Sample & Expression & Clinical application & Reference \\
\hline HOTAIR & ESCC & Tissue & Up & Unfavorable prognosis & [148] \\
\hline LOC285194 & ESCC & Tissue & Down & $\begin{array}{c}\text { Favorable prognosis and } \\
\text { prediction of response to } \\
\text { chemotherapy }\end{array}$ & {$[158]$} \\
\hline POU3F3 & ESCC & Plasma & Up & Diagnosis & {$[143]$} \\
\hline UCA1 & ESCC & Tissue & Up & Unfavorable prognosis & {$[151]$} \\
\hline SPRY4-IT1 & ESCC & Tissue & Up & Unfavorable prognosis & {$[152]$} \\
\hline PCAT-1 & ESCC & Tissue & Up & Unfavorable prognosis & {$[153]$} \\
\hline
\end{tabular}

A different study showed that increased HOTAIR expression is also associated with advanced TNM stage ESCC tumors and lymph node metastasis, a fact that strengthens its potential as a biomarker of poor prognosis [149]. Moreover, the association of a newly identified lncRNA FOXCUT with shorter survival time of patients after surgery, poorly differentiated tumors and metastasis, indicates its significant value as a biomarker of unfavorable prognosis of ESCC patients [150]. According to recent data, UCA1, a lncRNA with oncogenic role in ESCC, is an independent predictor of shorter OS and is related to advanced tumor stage and differentiation grade, increased lymphatic invasion as well as poor survival rates [151]. Two additional lncRNAs, SPRY4-IT1 and PCAT-1, may serve as indicators of unfavorable prognosis in ESCC since their expression levels were associated with markers of poor prognosis, such as lymphatic invasion, and shorter OS $[152,153]$. SPRY4-IT1 is also an independent prognostic marker of ESCC patients' OS [152]. Both PEG10 and TUG1 lncRNAs exhibit tumorigenic potential in EC, and recently they were found to be overexpressed in cancerous tissues compared to normal ones. It was also reported that expression levels of lncRNA PEG10 were positively correlated with lymph node metastasis and tumor differentiation status [154], whereas higher TUG1 expression levels were observed in patients with family history of EC as well as with upper segment of ESCC [155]. High PlncRNA-1 expression is found in advanced stage tumors (stage III/IV) and in tumors that spread to lymph nodes, suggesting that it may serve as a molecular marker of poor prognosis [156].

On the contrary, the expression of $91 \mathrm{H}$ is negatively associated with depth of tumor invasion, tumor grade, and TNM stage, demonstrating its favorable prognostic impact in ESCC [157]. Moreover, Tong et al. performed expression analysis of LOC285194 using ESCC tissue specimens from of patients prior to treatment, after chemoradiotherapy (CRT) and surgical resection, and from patients who underwent esophagectomy alone, in order to unravel the prognostic and predictive significance of this lncRNA. Interestingly, low LOC285194 expression was associated with adverse clinicopathological characteristics of the tumors such as TNM stage and distant metastasis. In the group of patients who received CRT, the expression of LOC285194 was found to be an independent predictor of patients' response to therapy, and in the subgroup patients after esophagectomy the LOC285194 levels were found to significantly correlate with their improved DFS and OS probabilities [158] (Table 3).

\section{IncRNAs as Therapeutic Modulators or Targets in Digestive System Malignancies}

The most prominent example of the utility of lncRNAs as therapeutic tools in targeted anticancer therapy is the construction of a DNA vector, namely, BC-819 (DTA-H19) which carries the diphtheria toxin A (DTA) gene under the control of transcriptional regulatory sequences of H19. The BC-819 plasmid takes advantage of the tumor specific expression of H19 and acts as a "trojan horse" to kill cancer cells through the expression of DTA [44]. The safety and efficiency of BC819 vector is evaluated in clinical trials and so far phase I/II studies have been completed in pancreatic cancer [40, 44].

The use of small interfering RNAs (siRNA) to inhibit lncRNAs is another approach with promising results regarding targeted therapy of GI cancers, although only in vitro data are available so far. In liver cancer cell lines, HOTAIR silencing inhibited cell proliferation and increased the cells sensitivity to Cisplatin and Doxorubicin [125]. MRUL (multidrug resistant-related and upregulated lncRNA) is overexpressed in two MDR GC cell sublines, and depletion of MRUL expression via siRNA, increased chemosensitivity of GC cells to Adriamycin and Vincristine [159]. Recently, a significant downregulation of snaR was observed in two 5fluorouracil (FU) resistant colon cancer cell lines (SNU-C4R and SNU-C5R) and siRNA silencing of this $\operatorname{lncRNA}$ resulted in increased cell viability after treatment with 5-FU [160].

Modulation of the expression of certain tumor-suppressor lncRNAs can be used as an alternative therapeutic approach in GI cancers. For instance, H19 upregulation suppressed MDR1 expression and sensitized the cells to Doxorubicin in vitro [161]. Furthermore, PVT1 overexpression in pancreatic cancer cells resulted in resistance to Gemcitabine [162]. Targeting of certain miRNA-lncRNA interaction axes holds promise as a therapeutic strategy in HCC and GC patients [163]. In particular, targeting of HOTAIR-miR-331$3 \mathrm{p}$-HER2 axis is a promising therapeutic perspective for HER-2 positive GC patients [68].

\section{Conclusions}

IncRNAs are steadily becoming one of the current trends in cancer research. In less than two years (2013-2015), an estimated total of $>700$ articles regarding the mechanistic and/or clinical role of lncRNAs in human malignancies has 
been published. It is expected that the profound implication of lncRNAs in cancer dynamics will be effectively translated into clinical practice through a novel class of biomarkers for major gastrointestinal malignancies. In fact, lncRNAs are endowed with certain characteristics that make them ideal as novel cancer biomarkers: (i) due to their broad implication in cancer pathobiology, a single deregulated lncRNA molecule can reflect changes in multiple cancer-affected pathways, (ii) they can be assayed in a wide variety of biological specimens, apart from tissues, such as serum or gastric fluid, where they show enhanced stability, (iii) they can be determined at low cost and can be easily assessed by simple methodologies such as qPCR that have already been introduced in routine clinical practice. Regarding the clinical management of GI cancers (Tables 1-3) some of the most promising lncRNAoriented studies include: (i) the development of a CCAT1specific peptide nucleic acid based molecular beacon for both imaging and in situ detection of CRC [82], (ii) the diagnostic value of HULC serum levels in HCC [123], (iii) the prognostic potential of HOTAIR for all GI cancers [86, 99, 103, 114, $127,136,149]$, and (iv) the predictive role of LOC285194 regarding chemoradiotherapy response in esophageal cancer [158]. However, there is an imperative need for validation of the biomarker potential of lncRNAs in GI malignancies, since up to now only the prognostic role of HOTAIR in GC $[101,103,114]$ and CRC $[65,85,86]$ has been demonstrated by at least two independent studies; in this respect, validation in prospective cohort studies could provide higher levels of evidence and accelerate the final clinical evaluation of specific lncRNAs as biomarkers.

Although the implication of lncRNAs in GI cancer is a fast expanding field of research, there are still many gaps that need to be filled. A further unraveling of the molecular functions of lncRNAs will significantly aid in the clarification of the genetic and molecular basis of cancer and is bound to provide novel lncRNA based therapeutics, apart from the ones currently being investigated in clinical trials for pancreatic cancer $[44,164]$. The elucidation of miRNA-mRNA-lncRNA axes of interaction, the deciphering of lncRNA gene methylation patterns, the characterization of SNPs affecting lncRNA expression levels, and/or function in GI malignancies could form the starting point in the identification of a novel therapeutic class of molecules for major digestive system malignancies.

\section{Abbreviations}

91H: $\quad$ Antisense H19

AFAP1: $\quad$ Actin filament associated protein 1

AFAP1-AS1: AFAP1 antisense RNA 1

AFP: $\quad$ Alpha-fetoprotein

AKAP-9: $\quad$ A kinase (PRKA) anchor protein 9

ANRIL: Antisense noncoding RNA in the INK4 locus

ASF1: $\quad$ Alternative splicing factor 1

BANCR: BRAF-activated non-protein coding RNA

CA19-9: Carbohydrate antigen 19-9

CARLo-5: Cancer-associated region long noncoding RNA
CCAT1: Colon cancer associated transcript 1

CCAT2: Colon cancer associated transcript 2

CDKN1A: Cyclin-dependent kinase inhibitor 1A

CRC: Colorectal cancer

CREB: $\quad$ cAMP response element-binding protein

CRNDE: Colorectal neoplasia differentially expressed

CRNDE-h: CRNDE transcript variant $h$

DFS: Disease-free survival

Dreh: $\quad$ Downregulated expression by $\mathrm{HBx}$

DSS: Disease-specific survival

DTA: Diphtheria toxin A

E2F1: $\quad$ E2F transcription factor 1

EAC: $\quad$ Esophageal adenocarcinoma

EC: $\quad$ Esophageal cancer

EMT: Epithelial-mesenchymal-transition

ESC: $\quad$ Embryonic stem cells

ESCC: $\quad$ Esophageal squamous cell carcinoma

EZH2: $\quad$ Enhancer of zeste 2 polycomb repressive complex 2 subunit

FDA: $\quad$ Food and Drug Administration

FENDRR: FOXF1 adjacent noncoding developmental regulatory RNA

FFPE: $\quad$ Formalin-fixed, paraffin-embedded (tissue)

FOXC1: $\quad$ Forkhead box C1

FOXCUT: FOXC1 upstream transcript

FU: $\quad$ Fluorouracil

GACAT1: Gastric cancer associated transcript 1

(previously known as AC096655.1-002)

GAS5: $\quad$ Growth arrest-specific 5

GC: $\quad$ Gastric cancer

GHET1: Gastric carcinoma proliferation enhancing transcript 1

GI: $\quad$ Gastrointestinal

GR: $\quad$ Glucocorticoid receptor

GRE: Glucocorticoid response elements

H19: $\quad$ Imprinted maternally expressed transcript

HBV: $\quad$ Hepatitis B virus

HBx: $\quad$ Hepatitis B virus $\mathrm{X}$ protein

HCC: Hepatocellular carcinoma

HDAC3: $\quad$ Histone deacetylase 3

HEIH: Hepatocellular carcinoma upregulated EZH2-associated long noncoding RNA

HIF-1a: Hypoxia-inducible factor 1, alpha subunit

HNF1A: Hepatocyte nuclear factor 1 homeobox A

HNF1A-AS1: HNF1A antisense RNA 1

HOTAIR: HOX transcript antisense intergenic RNA

HOTTIP: HOXA distal transcript antisense RNA

HOX: Homeobox

HULC: Highly upregulated in liver cancer

IGF2BP1: Insulin-like growth factor 2 mRNA

binding protein 1

Kcnqlotl: $\quad$ Kcnq1 overlapping transcript 1

LC: $\quad$ Liver cancer

LEIGC: Lower expression in gastric cancer

LET: Low expression in tumor

lincRNAs: Long intergenic RNA

lncRNAs: Long noncoding RNAs 
LSD1: Lysine-specific histone demethylase 1

MALAT1: Metastasis associated lung adenocarcinoma transcript 1

MAPK: $\quad$ Mitogen-activated protein kinase

MDR: $\quad$ Multiple drug resistance

MEG3: $\quad$ Maternally expressed 3

miRNA: MicroRNAs

MMP: $\quad$ Matrix metalloproteinase

MRUL: $\quad$ MDR-related and upregulated lncRNA

MVIH: Microvascular invasion in HCC

ncRAN: Noncoding RNA expressed in aggressive neuroblastoma

ncRuPAR: Nonprotein coding RNA, upstream of F2R/PAR1

NSCLC: Non-small cell lung carcinoma

ORFs: Open reading frames

OS: $\quad$ Overall survival

PANDA: $\quad$ P21 associated ncRNA DNA damage activated

PCA3: $\quad$ Prostate cancer associated 3

PCAT-1: $\quad$ Prostate cancer associated transcript 1

PCGEM1: Prostate-specific transcript 1

PCNA: Proliferating cell nuclear antigen

PDAC: Pancreatic ductal adenocarcinoma

PlncRNA-1: Prostate cancer-upregulated long noncoding RNA 1

POU3F3: $\quad$ POU class 3 homeobox 3

PRC2: $\quad$ Polycomb repression complex 2

PRKACB: cAMP-dependent protein kinase catalytic subunit b

PRNCR1: Prostate cancer associated noncoding RNA 1

PTBP2: Polypyrimidine tract binding protein 2

RB: $\quad$ Retinoblastoma

RBM38: $\quad$ RNA binding motif protein 38

RNAa: RNA activation

SCCA: Serum squamous cell carcinoma antigen

SF2: $\quad$ Pre-mRNA-splicing factor SF2

SFPQ: $\quad$ Splicing factor proline/glutamine-rich

siRNA: $\quad$ Small interfering RNA

SNPs:

SRA:

TCF7L2:

TERRA:

TGF- $\beta$ :

TNM:

T-UCR: Transcribed ultraconserved genomic region

TUG1: $\quad$ Taurine upregulated 1

UCA1: $\quad$ Urothelial cancer associated 1

URCH: Upregulated in hepatocellular carcinoma

VEGF: Vascular endothelial growth factor

XIST: $\quad \mathrm{X}$-inactive-specific transcript

Xlsirts: $\quad$ Xenopus laevis short interspersed repeat transcripts

ZEB1/2: $\quad$ Zinc finger E-box binding homeobox 1/2.

\section{Conflict of Interests}

The authors declare that there is no conflict of interests regarding the publication of this paper.

\section{References}

[1] J. Haringsma, N. C. M. van Heel, and E. J. Kuipers, "Are we making progress in diagnosing and preventing gastrointestinal cancers?" Therapeutic Advances in Gastroenterology, vol. 3, no. 4, pp. 213-220, 2010.

[2] J. Ferlay, I. Soerjomataram, R. Dikshit et al., "Cancer incidence and mortality worldwide: sources, methods and major patterns in GLOBOCAN 2012," International Journal of Cancer, vol. 136, no. 5, pp. E359-E386, 2015.

[3] Y. W. Sun, Y. F. Chen, J. Li et al., "A novel long non-coding RNA ENST00000480739 suppresses tumour cell invasion by regulating OS-9 and HIF- $1 \alpha$ in pancreatic ductal adenocarcinoma," British Journal of Cancer, vol. 111, no. 11, pp. 2131-2141, 2014.

[4] X. B. D'Journo and P. A. Thomas, "Current management of esophageal cancer," Journal of Thoracic Disease, vol. 6, supplement 2, pp. S253-S264, 2014.

[5] M. L. Rossi, A. A. Rehman, and C. S. Gondi, "Therapeutic options for the management of pancreatic cancer," World Journal of Gastroenterology, vol. 20, no. 32, pp. 11142-11159, 2014.

[6] S. Kasper and M. Schuler, "Targeted therapies in gastroesophageal cancer," European Journal of Cancer, vol. 50, no. 7, pp. 1247-1258, 2014.

[7] J. W. Shin and Y.-H. Chung, "Molecular targeted therapy for hepatocellular carcinoma: current and future," World Journal of Gastroenterology, vol. 19, no. 37, pp. 6144-6155, 2013.

[8] Y. Zheng, J. Zhou, and Y. Tong, "Gene signatures of drug resistance predict patient survival in colorectal cancer," Pharmacogenomics Journal, vol. 15, no. 2, pp. 135-143, 2014.

[9] M. Pertea, "The human transcriptome: an unfinished story," Genes, vol. 3, no. 3, pp. 344-360, 2012.

[10] J. Sana, P. Faltejskova, M. Svoboda, and O. Slaby, "Novel classes of non-coding RNAs and cancer," Journal of Translational Medicine, vol. 10, no. 1, article 103, 2012.

[11] S. A. Slavoff, A. J. Mitchell, A. G. Schwaid et al., "Peptidomic discovery of short open reading frame-encoded peptides in human cells," Nature Chemical Biology, vol. 9, no. 1, pp. 59-64, 2013.

[12] A. Gardini and R. Shiekhattar, "The many faces of long noncoding RNAs," The FEBS Journal, 2014.

[13] E. M. Reis and S. Verjovski-Almeida, "Perspectives of long noncoding RNAs in cancer diagnostics," Frontiers in Genetics, vol. 3, article 32, 2012.

[14] T. Gutschner and S. Diederichs, "The hallmarks of cancer: a long non-coding RNA point of view," RNA Biology, vol. 9, no. 6, pp. 703-709, 2012.

[15] E. A. Gibb, C. J. Brown, and W. L. Lam, "The functional role of long non-coding RNA in human carcinomas," Molecular Cancer, vol. 10, article 38, 2011.

[16] L. Ma, V. B. Bajic, and Z. Zhang, "On the classification of long non-coding RNAs,” RNA Biology, vol. 10, no. 6, pp. 925-933, 2013.

[17] C. I. Brannan, E. C. Dees, R. S. Ingram, and S. M. Tilghman, "The product of the H19 gene may function as an RNA," Molecular and Cellular Biology, vol. 10, no. 1, pp. 28-36, 1990. 
[18] C. J. Brown, A. Ballabio, J. L. Rupert et al., "A gene from the region of the human $\mathrm{X}$ inactivation centre is expressed exclusively from the inactive X chromosome," Nature, vol. 349, no. 6304, pp. 38-44, 1991.

[19] C. Xie, J. Yuan, H. Li et al., "NONCODEv4: exploring the world of long non-coding RNA genes," Nucleic Acids Research, vol. 42, no. 1, pp. D98-D103, 2014.

[20] O. Wapinski and H. Y. Chang, "Long noncoding RNAs and human disease," Trends in Cell Biology, vol. 21, no. 6, pp. 354361, 2011.

[21] M. Sun and W. Lee Kraus, "Minireview: long noncoding RNAs: new links between gene expression and cellular outcomes in endocrinology," Molecular Endocrinology, vol. 27, no. 9, pp. 1390-1402, 2013.

[22] J. R. Prensner and A. M. Chinnaiyan, "The emergence of lncRNAs in cancer biology," Cancer Discovery, vol. 1, no. 5, pp. 391-407, 2011.

[23] C. P. Ponting, P. L. Oliver, and W. Reik, "Evolution and functions of long noncoding RNAs," Cell, vol. 136, no. 4, pp. 629-641, 2009.

[24] E. S. Martens-Uzunova, R. Böttcher, C. M. Croce, G. Jenster, T. Visakorpi, and G. A. Calin, "Long noncoding RNA in prostate, bladder, and kidney cancer," European Urology, vol. 65, no. 6, pp. 1140-1151, 2014.

[25] A. Fatica and I. Bozzoni, "Long non-coding RNAs: new players in cell differentiation and development," Nature Reviews Genetics, vol. 15, no. 1, pp. 7-21, 2014.

[26] J. R. Alvarez-Dominguez, W. Hu, and H. F. Lodish, "Regulation of eukaryotic cell differentiation by long non-coding RNAs," in Molecular Biology of Long Non-coding RNAs, A. M. Khalil and J. Coller, Eds., pp. 15-67, Springer, New York, NY, USA, 2013.

[27] C. Wahlestedt, "Targeting long non-coding RNA to therapeutically upregulate gene expression," Nature Reviews Drug Discovery, vol. 12, no. 6, pp. 433-446, 2013.

[28] J. Li, Z. Xuan, and C. Liu, "Long non-coding RNAs and complex human diseases," International Journal of Molecular Sciences, vol. 14, no. 9, pp. 18790-18808, 2013.

[29] M. Hajjari, A. Khoshnevisan, and Y. K. Shin, "Molecular function and regulation of long non-coding RNAs: paradigms with potential roles in cancer," Tumor Biology, vol. 35, no. 11, pp. 10645-10663, 2014.

[30] R. A. Gupta, N. Shah, K. C. Wang et al., "Long non-coding RNA HOTAIR reprograms chromatin state to promote cancer metastasis," Nature, vol. 464, no. 7291, pp. 1071-1076, 2010.

[31] Z.-Y. Xu, Q.-M. Yu, Y.-A. Du et al., "Knockdown of long non-coding RNA HOTAIR suppresses tumor invasion and reverses epithelial-mesenchymal transition in gastric cancer," International Journal of Biological Sciences, vol. 9, no. 6, pp. 587597, 2013.

[32] J. T. Serviss, P. Johnsson, and D. Grandér, "An emerging role for long non-coding RNAs in cancer metastasis," Frontiers in Genetics, vol. 5, 2014.

[33] C. N. Niland, C. R. Merry, and A. M. Khalil, "Emerging roles for long non-coding RNAs in cancer and neurological disorders," Frontiers in Genetics, 2012.

[34] T. Kino, D. E. Hurt, T. Ichijo, N. Nader, and G. P. Chrousos, "Noncoding RNA Gas5 is a growth arrest- and starvationassociated repressor of the glucocorticoid receptor," Science Signaling, vol. 3, no. 107, p. ra8, 2010.

[35] G. L. Lee, A. Dobi, and S. Srivastava, "Prostate cancer: diagnostic performance of the PCA3 urine test," Nature Reviews Urology, vol. 8, no. 3, pp. 123-124, 2011.
[36] J. R. Day, M. Jost, M. A. Reynolds, J. Groskopf, and H. Rittenhouse, "PCA3: from basic molecular science to the clinical lab," Cancer Letters, vol. 301, no. 1, pp. 1-6, 2011.

[37] X.-S. Wang, Z. Zhang, H.-C. Wang et al., "Rapid identification of UCA1 as a very sensitive and specific unique marker for human bladder carcinoma," Clinical Cancer Research, vol. 12, no. 16, pp. 4851-4858, 2006.

[38] H. Zhang, Z. Chen, X. Wang, Z. Huang, Z. He, and Y. Chen, "Long non-coding RNA: a new player in cancer," Journal of Hematology and Oncology, vol. 6, no. 1, article 37, 2013.

[39] J. Fellenberg, L. Bernd, G. Delling, D. Witte, and A. ZahltenHinguranage, "Prognostic significance of drug-regulated genes in high-grade osteosarcoma," Modern Pathology, vol. 20, no. 10, pp. 1085-1094, 2007.

[40] M. C. Smaldone and B. J. Davies, "BC-819, a plasmid comprising the $\mathrm{H} 19$ gene regulatory sequences and diphtheria toxin $\mathrm{A}$, for the potential targeted therapy of cancers," Current Opinion in Molecular Therapeutics, vol. 12, no. 5, pp. 607-616, 2010.

[41] A. A. Sidi, P. Ohana, S. Benjamin et al., "Phase I/II marker lesion study of intravesical BC-819 DNA plasmid in H19 over expressing superficial bladder cancer refractory to bacillus calmette-guerin," Journal of Urology, vol. 180, no. 6, pp. 23792383, 2008.

[42] H. Ling, M. Fabbri, and G. A. Calin, "MicroRNAs and other non-coding RNAs as targets for anticancer drug development," Nature Reviews Drug Discovery, vol. 12, no. 11, pp. 847-865, 2013.

[43] F. Yang, X. Xue, L. Zheng et al., "Long non-coding RNA GHET1 promotes gastric carcinoma cell proliferation by increasing cMyc mRNA stability," FEBS Journal, vol. 281, no. 3, pp. 802-813, 2014.

[44] I. Matouk, E. Raveh, P. Ohana et al., “The increasing complexity of the oncofetal H19 gene locus: functional dissection and therapeutic intervention," International Journal of Molecular Sciences, vol. 14, no. 2, pp. 4298-4316, 2013.

[45] W. P. Tsang, E. K. O. Ng, S. S. M. Ng et al., "Oncofetal H19derived miR-675 regulates tumor suppressor RB in human colorectal cancer," Carcinogenesis, vol. 31, no. 3, pp. 350-358, 2010.

[46] F. Yang, J. Bi, X. Xue et al., "Up-regulated long non-coding RNA H19 contributes to proliferation of gastric cancer cells," FEBS Journal, vol. 279, no. 17, pp. 3159-3165, 2012.

[47] E.-B. Zhang, L. Han, D.-D. Yin, R. Kong, W. De, and J. Chen, "c-Myc-induced, long, noncoding H19 affects cell proliferation and predicts a poor prognosis in patients with gastric cancer," Medical Oncology, vol. 31, article 914, 2014.

[48] X. He, X. Tan, X. Wang et al., "C-Myc-activated long noncoding RNA CCAT1 promotes colon cancer cell proliferation and invasion," Tumor Biology, vol. 35, no. 12, pp. 12181-12188, 2014.

[49] T. Kim, R. Cui, Y.-J. Jeon et al., "Long-range interaction and correlation between MYC enhancer and oncogenic long noncoding RNA CARLo-5," Proceedings of the National Academy of Sciences of the United States of America, vol. 111, no. 11, pp. 4173-4178, 2014.

[50] Y. Zhang, M. Ma, W. Liu, W. Ding, and H. Yu, "Enhanced expression of long noncoding RNA CARLo-5 is associated with the development of gastric cancer," International Journal of Clinical \& Experimental Pathology, vol. 7, no. 12, pp. 8471-8479, 2014.

[51] F. Yang, L. Zhang, X.-S. Huo et al., "Long noncoding RNA high expression in hepatocellular carcinoma facilitates tumor growth through enhancer of zeste homolog 2 in humans," Hepatology, vol. 54, no. 5, pp. 1679-1689, 2011. 
[52] K. Panzitt, M. M. O. Tschernatsch, C. Guelly et al., "Characterization of HULC, a novel gene with striking up-regulation in hepatocellular carcinoma, as noncoding RNA," Gastroenterology, vol. 132, no. 1, pp. 330-342, 2007.

[53] Y. Du, G. Kong, X. You et al., "Elevation of highly up-regulated in liver cancer (HULC) by hepatitis B virus X protein promotes hepatoma cell proliferation via down-regulating p18," Journal of Biological Chemistry, vol. 287, no. 31, pp. 26302-26311, 2012.

[54] J. Wang, X. Liu, H. Wu et al., "CREB up-regulates long non-coding RNA, HULC expression through interaction with microRNA-372 in liver cancer," Nucleic Acids Research, vol. 38, no. 16, pp. 5366-5383, 2010.

[55] Y. Takahashi, G. Sawada, J. Kurashige et al., "Amplification of PVT-1 is involved in poor prognosis via apoptosis inhibition in colorectal cancers," British Journal of Cancer, vol. 110, no. 1, pp. 164-171, 2014.

[56] J. Wang, L. Su, X. Chen et al., "MALAT1 promotes cell proliferation in gastric cancer by recruiting SF2/ASF," Biomedicine and Pharmacotherapy, vol. 68, no. 5, pp. 557-564, 2014.

[57] M. Sun, F.-Y. Jin, R. Xia et al., "Decreased expression of long noncoding RNA GAS5 indicates a poor prognosis and promotes cell proliferation in gastric cancer," BMC Cancer, vol. 14, no. 1, article 319, 2014.

[58] X. Lu, Y. Fang, Z. Wang et al., "Downregulation of gas5 increases pancreatic cancer cell proliferation by regulating CDK6," Cell and Tissue Research, vol. 354, no. 3, pp. 891-896, 2013.

[59] M. Sun, R. Xia, F. Jin et al., "Downregulated long noncoding RNA MEG3 is associated with poor prognosis and promotes cell proliferation in gastric cancer," Tumor Biology, vol. 35, no. 2, pp. 1065-1073, 2014.

[60] D. Yin, Z. Liu, E. Zhang, R. Kong, Z. Zhang, and R. Guo, "Decreased expression of long noncoding RNA MEG3 affects cell proliferation and predicts a poor prognosis in patients with colorectal cancer," Tumor Biology, 2015.

[61] Y. Zhou, Y. Zhong, Y. Wang et al., "Activation of p53 by MEG3 non-coding RNA," The Journal of Biological Chemistry, vol. 282, no. 34, pp. 24731-24742, 2007.

[62] C. Braconi, T. Kogure, N. Valeri et al., "MicroRNA-29 can regulate expression of the long non-coding RNA gene MEG3 in hepatocellular cancer," Oncogene, vol. 30, no. 47, pp. 4750-4756, 2011.

[63] J. Yan, X. Guo, J. Xia et al., "MiR-148a regulates MEG3 in gastric cancer by targeting DNA methyltransferase 1," Medical Oncology, vol. 31, no. 3, article 879, 2014.

[64] Q. Liu, J. Huang, N. Zhou et al., "LncRNA loc285194 is a p53regulated tumor suppressor," Nucleic Acids Research, vol. 41, no. 9, pp. 4976-4987, 2013.

[65] R. Kogo, T. Shimamura, K. Mimori et al., "Long noncoding RNA HOTAIR regulates polycomb-dependent chromatin modification and is associated with poor prognosis in colorectal cancers," Cancer Research, vol. 71, no. 20, pp. 6320-6326, 2011.

[66] C. Ding, S. Cheng, Z. Yang et al., "Long non-coding RNA HOTAIR promotes cell migration and invasion via downregulation of RNA binding motif protein 38 in hepatocellular carcinoma cells," International Journal of Molecular Sciences, vol. 15, no. 3, pp. 4060-4076, 2014.

[67] Y. J. Geng, S. L. Xie, Q. Li, J. Ma, and G. Y. Wang, "Large intervening non-coding RNA HOTAIR is associated with hepatocellular carcinoma progression," Journal of International Medical Research, vol. 39, no. 6, pp. 2119-2128, 2011.
[68] X.-H. Liu, M. Sun, F.-Q. Nie et al., "Lnc RNA HOTAIR functions as a competing endogenous RNA to regulate HER2 expression by sponging miR-331-3p in gastric cancer," Molecular Cancer, vol. 13, no. 1, article 92, 2014.

[69] M. Z. Ma, C. X. Li, Y. Zhang et al., "Long non-coding RNA HOTAIR, a c-Myc activated driver of malignancy, negatively regulates miRNA-130a in gallbladder cancer," Molecular Cancer, vol. 13, no. 1, article 156, 2014.

[70] Q. Ji, L. Zhang, X. Liu et al., "Long non-coding RNA MALAT1 promotes tumour growth and metastasis in colorectal cancer through binding to SFPQ and releasing oncogene PTBP2 from SFPQ/PTBP2 complex," British Journal of Cancer, vol. 111, no. 4, pp. 736-748, 2014.

[71] M. H. Yang, Z. Y. Hu, C. Xu et al., "MALAT1 promotes colorectal cancer cell proliferation/migration/invasion via PRKA kinase anchor protein 9," Biochimica et Biophysica Acta-Molecular Basis of Disease, vol. 1852, no. 1, pp. 166-174, 2015.

[72] H. Ling, R. Spizzo, Y. Atlasi et al., "CCAT2, a novel noncoding RNA mapping to 8q24, underlies metastatic progression and chromosomal instability in colon cancer," Genome Research, vol. 23, no. 9, pp. 1446-1461, 2013.

[73] I. J. Matouk, N. DeGroot, S. Mezan et al., “The H19 non-coding RNA is essential for human tumor growth," PLoS ONE, vol. 2, no. 9, article e845, 2007.

[74] L. Zhang, F. Yang, J.-H. Yuan et al., "Epigenetic activation of the MiR-200 family contributes to H19-mediated metastasis suppression in hepatocellular carcinoma," Carcinogenesis, vol. 34, no. 3, pp. 577-586, 2013.

[75] F. Yang, X.-S. Huo, S.-X. Yuan et al., "Repression of the long noncoding RNA-LET by histone deacetylase 3 contributes to hypoxia-mediated metastasis," Molecular Cell, vol. 49, no. 6, pp. 1083-1096, 2013.

[76] J. F. Huang, Y. J. Guo, C. X. Zhao et al., "Hepatitis B virus X protein $(\mathrm{HBx})$-related long noncoding RNA (lncRNA) downregulated expression by $\mathrm{HBx}$ (Dreh) inhibits hepatocellular carcinoma metastasis by targeting the intermediate filament protein vimentin," Hepatology, vol. 57, no. 5, pp. 1882-1892, 2013.

[77] Y. Han, J. Ye, D. Wu et al., "LEIGC long non-coding RNA acts as a tumor suppressor in gastric carcinoma by inhibiting the epithelial-to-mesenchymal transition," BMC Cancer, vol. 14, no. 1, p. 932, 2014.

[78] L. D. Graham, S. K. Pedersen, G. S. Brown et al., "Colorectal neoplasia differentially expressed (CRNDE), a novel gene with elevated expression in colorectal adenomas and adenocarcinomas," Genes and Cancer, vol. 2, no. 8, pp. 829-840, 2011.

[79] B. Yan, W. Gu, Z. Yang et al., "Downregulation of a long noncoding RNA-ncRuPAR contributes to tumor inhibition in colorectal cancer," Tumor Biology, vol. 35, no. 11, pp. 11329-11335, 2014.

[80] A. Nissan, A. Stojadinovic, S. Mitrani-Rosenbaum et al., "Colon cancer associated transcript-1: a novel RNA expressed in malignant and pre-malignant human tissues," International Journal of Cancer, vol. 130, no. 7, pp. 1598-1606, 2012.

[81] B. Alaiyan, N. Ilyayev, A. Stojadinovic et al., "Differential expression of colon cancer associated transcriptl (CCAT1) along the colonic adenoma-carcinoma sequence," BMC Cancer, vol. 13, article 196, 2013.

[82] Y. Kam, A. Rubinstein, S. Naik et al., "Detection of a long non-coding RNA (CCAT1) in living cells and human adenocarcinoma of colon tissues using FIT-PNA molecular beacons," Cancer Letters, vol. 352, no. 1, pp. 90-96, 2014. 
[83] L. Li, R. Sun, Y. Liang et al., "Association between polymorphisms in long non-coding RNA PRNCR1 in 8q24 and risk of colorectal cancer," Journal of Experimental and Clinical Cancer Research, vol. 32, no. 1, article 104, 2013.

[84] Y. Xue, D. Gu, G. Ma et al., "Genetic variants in lncRNA HOTAIR are associated with risk of colorectal cancer," Mutagenesis, vol. 30, no. 2, pp. 303-310, 2015.

[85] Z. Wu, X. Wang, H. Tang et al., "Long non-coding RNA HOTAIR is a powerful predictor of metastasis and poor prognosis and is associated with epithelial-mesenchymal transition in colon cancer," Oncology Reports, vol. 32, no. 1, pp. 395-402, 2014.

[86] M. Svoboda, J. Slyskova, M. Schneiderova et al., "HOTAIR long non-coding RNA is a negative prognostic factor not only in primary tumors, but also in the blood of colorectal cancer patients," Carcinogenesis, vol. 35, no. 7, pp. 1510-1515, 2014.

[87] H.-T. Zheng, D.-B. Shi, Y.-W. Wang et al., "High expression of lncRNA MALAT1 suggests a biomarker of poor prognosis in colorectal cancer," International Journal of Clinical \& Experimental Pathology, vol. 7, no. 6, pp. 3174-3181, 2014.

[88] Y. Han, Y. N. Yang, H. H. Yuan et al., "UCA1, a long noncoding RNA up-regulated in colorectal cancer influences cell proliferation, apoptosis and cell cycle distribution," Pathology, vol. 46, no. 5, pp. 396-401, 2014.

[89] X. Ge, Y. Chen, X. Liao et al., "Overexpression of long noncoding RNA PCAT-1 is a novel biomarker of poor prognosis in patients with colorectal cancer," Medical Oncology, vol. 30, no. 2, article 588, 2013.

[90] Q. Deng, B. He, T. Gao et al., "Up-regulation of $91 \mathrm{H}$ promotes tumor metastasis and predicts poor prognosis for patients with colorectal cancer," PLoS ONE, vol. 9, no. 7, Article ID e103022, 2014.

[91] Q. Guo, Y. Zhao, J. Chen et al., "BRAF-activated long noncoding RNA contributes to colorectal cancer migration by inducing epithelial-mesenchymal transition," Oncology Letters, vol. 8, no. 2, pp. 869-875, 2014.

[92] M. D. Xu, P. Qi, W. W. Weng et al., "Long non-coding RNA LSINCT5 predicts negative prognosis and exhibits oncogenic activity in gastric cance," Medicine, vol. 93, no. 28, article e303, 2014.

[93] P. Qi, M.-D. Xu, S.-J. Ni et al., "Low expression of LOC285194 is associated with poor prognosis in colorectal cancer," Journal of Translational Medicine, vol. 11, no. 1, article 122, 2013.

[94] P. Qi, M.-D. Xu, S.-J. Ni et al., "Down-regulation of ncRAN, a long non-coding RNA, contributes to colorectal cancer cell migration and invasion and predicts poor overall survival for colorectal cancer patients," Molecular Carcinogenesis, 2014.

[95] D. Shi, H. Zheng, C. Zhuo et al., "Low expression of novel lncRNA RP11-462C24.1 suggests a biomarker of poor prognosis in colorectal cancer," Medical Oncology, vol. 31, no. 7, p. 31, 2014.

[96] J. Sana, S. Hankeova, M. Svoboda, I. Kiss, R. Vyzula, and O. Slaby, "Expression levels of transcribed ultraconserved regions uc.73 and uc.388 are altered in colorectal cancer," Oncology, vol. 82, no. 2, pp. 114-118, 2012.

[97] D. Yin, X. He, E. Zhang, R. Kong, W. De, and Z. Zhang, "Long noncoding RNA GAS5 affects cell proliferation and predicts a poor prognosis in patients with colorectal cancer," Medical Oncology, vol. 31, no. 11, article 253, 2014.

[98] Y. Hu, H.-Y. Chen, C.-Y. Yu et al., "A long non-coding RNA signature to improve prognosis prediction of colorectal cancer," Oncotarget, vol. 5, no. 8, pp. 2230-2242, 2014.
[99] Y. Okugawa, Y. Toiyama, K. Hur et al., "Metastasis-associated long non-coding RNA drives gastric cancer development and promotes peritoneal metastasis," Carcinogenesis, vol. 35, no. 12, pp. 2731-2739, 2014.

[100] N. K. Lee, J. H. Lee, C. H. Park et al., "Long non-coding RNA HOTAIR promotes carcinogenesis and invasion of gastric adenocarcinoma," Biochemical and Biophysical Research Communications, vol. 451, no. 2, pp. 171-178, 2014.

[101] E. Emadi-Andani, P. Nikpour, M. Emadi-Baygi, and A. Bidmeshkipour, "Association of HOTAIR expression in gastric carcinoma with invasion and distant metastasis," Advanced Biomedical Research, vol. 3, no. 1, article 135, 2014.

[102] H. Endo, T. Shiroki, T. Nakagawa et al., "Enhanced expression of long non-coding RNA HOTAIR is associated with the development of gastric cancer," PLoS ONE, vol. 8, no. 10, Article ID e77070, 2013.

[103] M. Hajjari, M. Behmanesh, M. Sadeghizadeh, and M. Zeinoddini, "Up-regulation of HOTAIR long non-coding RNA in human gastric adenocarcinoma tissues," Medical Oncology, vol. 30, no. 3, article 670, 2013.

[104] H. Li, B. Yu, J. Li et al., "Overexpression of lncRNA H19 enhances carcinogenesis and metastasis of gastric cancer," Oncotarget, vol. 5, no. 8, pp. 2318-2329, 2014.

[105] E.-B. Zhang, R. Kong, D.-D. Yin et al., "Long noncoding RNA ANRIL indicates a poor prognosis of gastric cancer and promotes tumor growth by epigenetically silencing of miR99a/miR-449a," Oncotarget, vol. 5, no. 8, pp. 2276-2292, 2014.

[106] Y. Zhao, Q. Guo, J. Chen, J. Hu, S. Wang, and Y. Sun, "Role of long non-coding RNA HULC in cell proliferation, apoptosis and tumor metastasis of gastric cancer: a clinical and in vitro investigation," Oncology Reports, vol. 31, no. 1, pp. 358-364, 2014.

[107] X. Lin, M. Yang, T. Xia, and J. Guo, "Increased expression of long noncoding RNA ABHD11-AS1 in gastric cancer and its clinical significance," Medical Oncology, vol. 31, article 42, 2014.

[108] J. Ding, D. Li, M. Gong et al., "Expression and clinical significance of the long non-coding RNA PVT1 in human gastric cancer," OncoTargets and Therapy, vol. 2014, article 7, pp. 16251630, 2014

[109] X. Chen, J. Sun, Y. Song et al., "The novel long noncoding RNA AC138128.1 may be a predictive biomarker in gastric cancer," Medical Oncology, vol. 31, no. 11, p. 262, 2014.

[110] Y. Shao, M. Ye, X. Jiang et al., "Gastric juice long noncoding RNA used as a tumor marker for screening gastric cancer," Cancer, vol. 120, no. 21, pp. 3320-3328, 2014.

[111] T. P. Xu, M. D. Huang, R. Xia et al., "Decreased expression of the long non-coding RNA FENDRR is associated with poor prognosis in gastric cancer and FENDRR regulates gastric cancer cell metastasis by affecting fibronectinl expression," Journal of Hematology \& Oncology, vol. 7, no. 1, article 63, 2014.

[112] W. Sun, Y. Wu, X. Yu et al., "Decreased expression of long noncoding RNA AC096655.1-002 in gastric cancer and its clinical significance," Tumor Biology, vol. 34, no. 5, pp. 26972701, 2013.

[113] Z. Liu, Y. Shao, L. Tan, H. Shi, S. Chen, and J. Guo, "Clinical significance of the low expression of FER1L4 in gastric cancer patients," Tumor Biology, vol. 35, no. 10, pp. 9613-9617, 2014.

[114] T. Arita, D. Ichikawa, H. Konishi et al., "Circulating long non-coding RNAs in plasma of patients with gastric cancer," Anticancer Research, vol. 33, no. 8, pp. 3185-3193, 2013.

[115] Q. Pang, J. Ge, Y. Shao et al., "Increased expression of long intergenic non-coding RNA LINC00152 in gastric cancer and 
its clinical significance," Tumor Biology, vol. 35, no. 6, pp. 54415447, 2014.

[116] W.-J. Cao, H.-L. Wu, B.-S. He, Y.-S. Zhang, and Z.-Y. Zhang, "Analysis of long non-coding RNA expression profiles in gastric cancer," World Journal of Gastroenterology, vol. 19, no. 23, pp. 3658-3664, 2013.

[117] H. Song, W. Sun, G. Ye et al., "Long non-coding RNA expression profile in human gastric cancer and its clinical significances," Journal of Translational Medicine, vol. 11, no. 1, p. 225, 2013.

[118] I. Mizrahi, H. Mazeh, R. Grinbaum et al., "Colon cancer associated transcript-1 (CCAT1) expression in adenocarcinoma of the stomach," Journal of Cancer, vol. 6, no. 2, pp. 105-110, 2015.

[119] F. Yang, X. Xue, J. Bi et al., "Long noncoding RNA CCAT1, which could be activated by c-Myc, promotes the progression of gastric carcinoma," Journal of Cancer Research and Clinical Oncology, vol. 139, no. 3, pp. 437-445, 2013.

[120] C. Y. Wang, L. Hua, K. H. Yao, J. T. Chen, J. J. Zhang, and J. $\mathrm{H}$. Hu, "Long non-coding RNA CCAT2 is up-regulated in gastric cancer and associated with poor prognosis," International Journal of Clinical and Experimental Pathology, vol. 8, no. 1, pp. 779-785, 2015.

[121] B. Zhou, X.-Y. Jing, J.-Q. Wu, H.-F. Xi, and G.-J. Lu, "Downregulation of long non-coding RNA LET is associated with poor prognosis in gastric cancer," International Journal of Clinical \& Experimental Patholog, vol. 7, no. 12, pp. 8893-8898, 2014.

[122] S.-M. Park, S.-J. Park, H.-J. Kim et al., "A known expressed sequence tag, BM742401, is a potent lincRNA inhibiting cancer metastasis," Experimental and Molecular Medicine, vol. 45, no. 7, article e31, 2013.

[123] H. Xie, H. Ma, and D. Zhou, "Plasma HULC as a promising novel biomarker for the detection of hepatocellular carcinoma," BioMed Research International, vol. 2013, Article ID 136106, 5 pages, 2013.

[124] J. Lu, F. Xie, L. Geng, W. Shen, C. Sui, and J. Yang, "Investigation of serum lncRNA-uc003wbd and lncRNA-AF085935 expression profile in patients with hepatocellular carcinoma and HBV," Tumor Biology, 2014.

[125] J. Zhao, C. M. Greene, S. G. Gray, and M. W. Lawless, "Long noncoding RNAs in liver cancer: what we know in 2014," Expert Opinion on Therapeutic Targets, vol. 18, no. 10, pp. 1207-1218, 2014.

[126] J. Zhu, S. Liu, F. Ye et al., "The long noncoding RNA expression profile of hepatocellular carcinoma identified by microarray analysis," PLoS ONE, vol. 9, no. 7, Article ID e101707, 2014.

[127] M. Ishibashi, R. Kogo, K. Shibata et al., "Clinical significance of the expression of long non-coding RNA HOTAIR in primary hepatocellular carcinoma," Oncology Reports, vol. 29, no. 3, pp. 946-950, 2013.

[128] S.-X. Yuan, F. Yang, Y. Yang et al., "Long noncoding RNA associated with microvascular invasion in hepatocellular carcinoma promotes angiogenesis and serves as a predictor for hepatocellular carcinoma patients' poor recurrence-free survival after hepatectomy," Hepatology, vol. 56, no. 6, pp. 2231-2241, 2012.

[129] W. H. Xu, J. B. Zhang, Z. Dang et al., "Long non-coding RNA URHC regulates cell proliferation and apoptosis via ZAK through the ERK/MAPK signaling pathway in hepatocellular carcinoma," International Journal of Biological Sciences, vol. 10, no. 7, pp. 664-676, 2014.

[130] J.-H. Yuan, F. Yang, F. Wang et al., "A long noncoding RNA Activated by TGF- $\beta$ promotes the invasion-metastasis cascade in hepatocellular carcinoma," Cancer Cell, vol. 25, no. 5, pp. 666681, 2014.
[131] L. Quagliata, M. S. Matter, S. Piscuoglio et al., "Long noncoding RNA HOTTIP/HOXA13 expression is associated with disease progression and predicts outcome in hepatocellular carcinoma patients," Hepatology, vol. 59, no. 3, pp. 911-923, 2014.

[132] C. Ding, Z. Yang, Z. Lv et al., "Long non-coding RNA PVT1 is associated with tumor progression and predicts recurrence in hepatocellular carcinoma patients," Oncology Letters, vol. 9, no. 2, pp. 955-963, 2015.

[133] Z.-Q. Tu, R.-J. Li, J.-Z. Mei, and X.-H. Li, “Down-regulation of long non-coding RNA GAS5 is associated with the prognosis of hepatocellular carcinoma," International Journal of Clinical \& Experimental Pathology, vol. 7, no. 7, pp. 4303-4309, 2014.

[134] J.-H. Liu, G. Chen, Y.-W. Dang, C.-J. Li, and D.-Z. Luo, "Expression and prognostic significance of lncRNA MALAT1 in pancreatic cancer tissues," Asian Pacific Journal of Cancer Prevention, vol. 15, no. 7, pp. 2971-2977, 2014.

[135] E. J. Pang, R. Yang, X. B. Fu, and Y. F. Liu, "Overexpression of long non-coding RNA MALAT1 is correlated with clinical progression and unfavorable prognosis in pancreatic cancer," Tumor Biology, vol. 36, no. 4, pp. 2403-2407, 2015.

[136] K. Kim, I. Jutooru, G. Chadalapaka et al., "HOTAIR is a negative prognostic factor and exhibits pro-oncogenic activity in pancreatic cancer," Oncogene, vol. 32, no. 13, pp. 1616-1625, 2013.

[137] C. S. Huang, W. Yu, H. Cui et al., "Increased expression of the lncRNA PVT1 is associated with poor prognosis in pancreatic cancer patients," Minerva Medica, vol. 10, p. 10, 2015.

[138] J. Li, D. Liu, R. Hua et al., "Long non-coding RNAs expressed in pancreatic ductal adenocarcinoma and lncRNA BC008363 an independent prognostic factor in PDAC," Pancreatology, vol. 14, no. 5, pp. 385-390, 2014.

[139] A. C. Tahira, M. S. Kubrusly, M. F. Faria et al., "Long noncoding intronic RNAs are differentially expressed in primary and metastatic pancreatic cancer," Molecular Cancer, vol. 10, article 141, 2011.

[140] C. Ma, K. Nong, H. Zhu et al., "H19 promotes pancreatic cancer metastasis by derepressing let-7's suppression on its target HMGA2-mediated EMT,' Tumor Biology, vol. 35, no. 9, pp. 9163-9169, 2014.

[141] D. Chen, Z. Zhang, C. Mao et al., "ANRIL inhibits p15INK4b through the TGF $\beta 1$ signaling pathway in human esophageal squamous cell carcinoma," Cellular Immunology, vol. 289, no. 1-2, pp. 91-96, 2014.

[142] W. Li, J. Zheng, J. Deng et al., "Increased levels of the long intergenic non-protein coding RNA POU3F3 promote DNA methylation in esophageal squamous cell carcinoma cells," Gastroenterology, vol. 146, no. 7, pp. 1714.e5-1726.e5, 2014.

[143] Y. S. Tong, X. W. Wang, X. L. Zhou et al., "Identification of the long non-coding RNA POU3F3 in plasma as a novel biomarker for diagnosis of esophageal squamous cell carcinoma," Molecular Cancer, vol. 14, no. 1, article 3, 2015.

[144] H. Wu, J. Zheng, J. Deng et al., "A genetic polymorphism in lincRNA-uc003opf.1 is associated with susceptibility to esophageal squamous cell carcinoma in Chinese populations," Carcinogenesis, vol. 34, no. 12, pp. 2908-2917, 2013.

[145] X. Yang, J. H. Song, Y. Cheng et al., "Long non-coding RNA HNF1A-AS1 regulates proliferation and migration in oesophageal adenocarcinoma cells," Gut, vol. 63, no. 6, pp. 881890, 2014.

[146] W. Wu, T. D. Bhagat, X. Yang et al., "Hypomethylation of noncoding DNA regions and overexpression of the long noncoding RNA, AFAP1-AS1, in Barrett's esophagus and esophageal 
adenocarcinoma," Gastroenterology, vol. 144, no. 5, pp. 956.e4966.e4, 2013.

[147] M. Fassan, L. Dall'Olmo, M. Galasso et al., "Transcribed ultraconserved noncoding RNAs (T-UCR) are involved in Barrett's esophagus carcinogenesis," Oncotarget, vol. 5, no. 16, pp. 71627171, 2014.

[148] X.-S. Ge, H.-J. Ma, X.-H. Zheng et al., "HOTAIR, a prognostic factor in esophageal squamous cell carcinoma, inhibits WIF-1 expression and activates Wnt pathway," Cancer Science, vol. 104, no. 12, pp. 1675-1682, 2013.

[149] F. Chen, M. Sun, S. Li et al., "Upregulation of the long non-coding rna hotair promotes esophageal squamous cell carcinoma metastasis and poor prognosis," Molecular Carcinogenesis, vol. 52, no. 11, pp. 908-915, 2013.

[150] F. Pan, J. Yao, Y. Chen et al., "A novel long non-coding RNA FOXCUT and mRNA FOXC1 pair promote progression and predict poor prognosis in esophageal squamous cell carcinoma," International Journal of Clinical \& Experimental Pathology, vol. 7, no. 6, pp. 2838-2849, 2014.

[151] J. Y. Li, X. Ma, and C. B. Zhang, "Overexpression of long noncoding RNA UCA1 predicts a poor prognosis in patients with esophageal squamous cell carcinoma," International Journal of Clinical and Experimental Pathology, vol. 7, no. 11, pp. 79387944, 2014.

[152] H.-W. Xie, Q.-Q. Wu, B. Zhu et al., "Long noncoding RNA SPRY4-IT1 is upregulated in esophageal squamous cell carcinoma and associated with poor prognosis," Tumor Biology, vol. 35, no. 8, pp. 7743-7754, 2014.

[153] W.-H. Shi, Q.-Q. Wu, S.-Q. Li et al., "Upregulation of the long noncoding RNA PCAT-1 correlates with advanced clinical stage and poor prognosis in esophageal squamous carcinoma," Tumor Biology, vol. 36, no. 4, pp. 2501-2507, 2015.

[154] W. Zang, T. Wang, J. Huang et al., "Long noncoding RNA PEG10 regulates proliferation and invasion of esophageal cancer cells," Cancer Gene Therapy, vol. 22, no. 3, pp. 138-144, 2015.

[155] Y. Xu, J. Wang, M. Qiu et al., "Upregulation of the long noncoding RNA TUG1 promotes proliferation and migration of esophageal squamous cell carcinoma," Tumor Biology, vol. 36, no. 3, pp. 1643-1651, 2015.

[156] C. Wang, Q. Wu, S. Li et al., "Upregulation of the long noncoding RNA PlncRNA-1 promotes esophageal squamous carcinoma cell proliferation and correlates with advanced clinical stage," Digestive Diseases and Sciences, vol. 59, no. 3, pp. 591-597, 2014.

[157] T. Gao, B. He, Y. Pan et al., "Long non-coding RNA 91H contributes to the occurrence and progression of esophageal squamous cell carcinoma by inhibiting IGF2 expression," Molecular Carcinogenesis, vol. 54, no. 5, pp. 359-367, 2015.

[158] Y. S. Tong, X. L. Zhou, X. W. Wang et al., "Association of decreased expression of long non-coding RNA LOC285194 with chemoradiotherapy resistance and poor prognosis in esophageal squamous cell carcinoma," Journal of Translational Medicine, vol. 12, article 233, 2014.

[159] Y. Wang, D. Zhang, K. Wu, Q. Zhao, Y. Nie, and D. Fan, "Long noncoding RNA MRUL promotes ABCB1 expression in multidrug-resistant gastric cancer cell sublines," Molecular and Cellular Biology, vol. 34, no. 17, pp. 3182-3193, 2014.

[160] H. Lee, C. Kim, J. L. Ku et al., "A long non-coding RNA snaR contributes to 5 -fluorouracil resistance in human colon cancer cells," Molecules and Cells, vol. 37, no. 7, pp. 540-546, 2014.
[161] W. P. Tsang and T. T. Kwok, "Riboregulator H19 induction of MDR1-associated drug resistance in human hepatocellular carcinoma cells," Oncogene, vol. 26, no. 33, pp. 4877-4881, 2007.

[162] L. You, D. Chang, H.-Z. Du, and Y.-P. Zhao, "Genome-wide screen identifies PVT1 as a regulator of Gemcitabine sensitivity in human pancreatic cancer cells," Biochemical and Biophysical Research Communications, vol. 407, no. 1, pp. 1-6, 2011.

[163] Y. He, X.-M. Meng, C. Huang et al., "Long noncoding RNAs: novel insights into hepatocelluar carcinoma," Cancer Letters, vol. 344, no. 1, pp. 20-27, 2014.

[164] N. Hanna, P. Ohana, F. M. Konikoff et al., "Phase 1/2a, doseescalation, safety, pharmacokinetic and preliminary efficacy study of intratumoral administration of BC-819 in patients with unresectable pancreatic cancer," Cancer Gene Therapy, vol. 19, no. 6, pp. 374-381, 2012. 


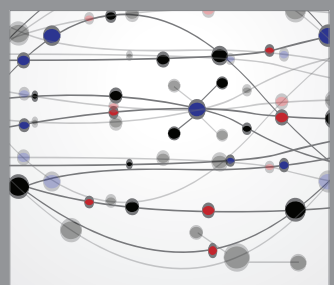

The Scientific World Journal
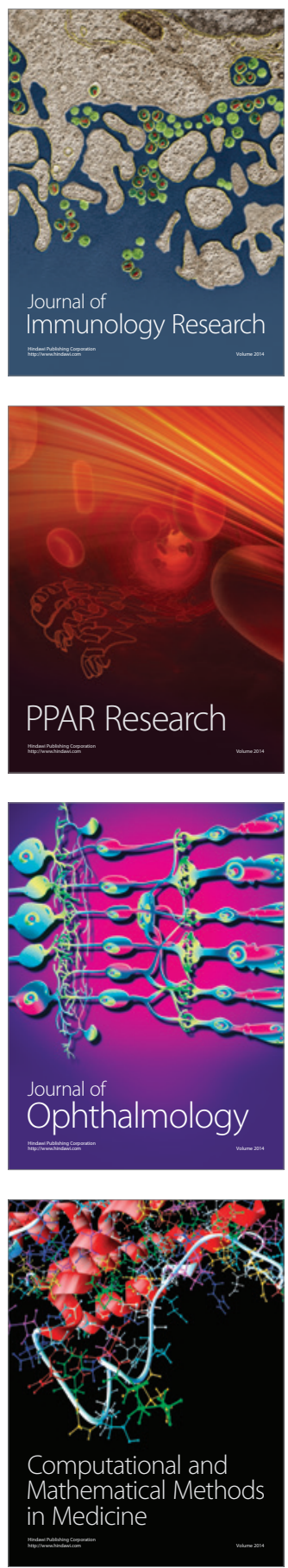

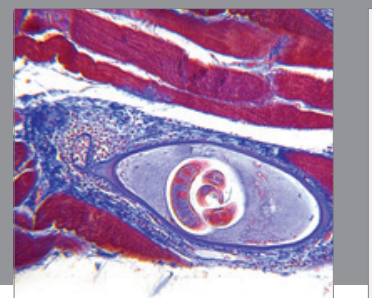

Gastroenterology

Research and Practice
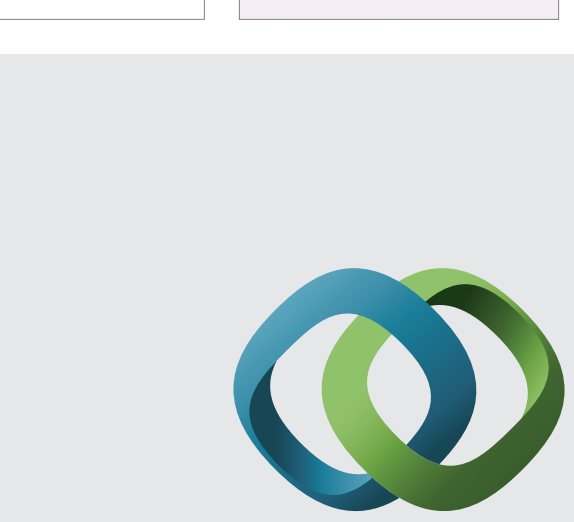

\section{Hindawi}

Submit your manuscripts at

http://www.hindawi.com
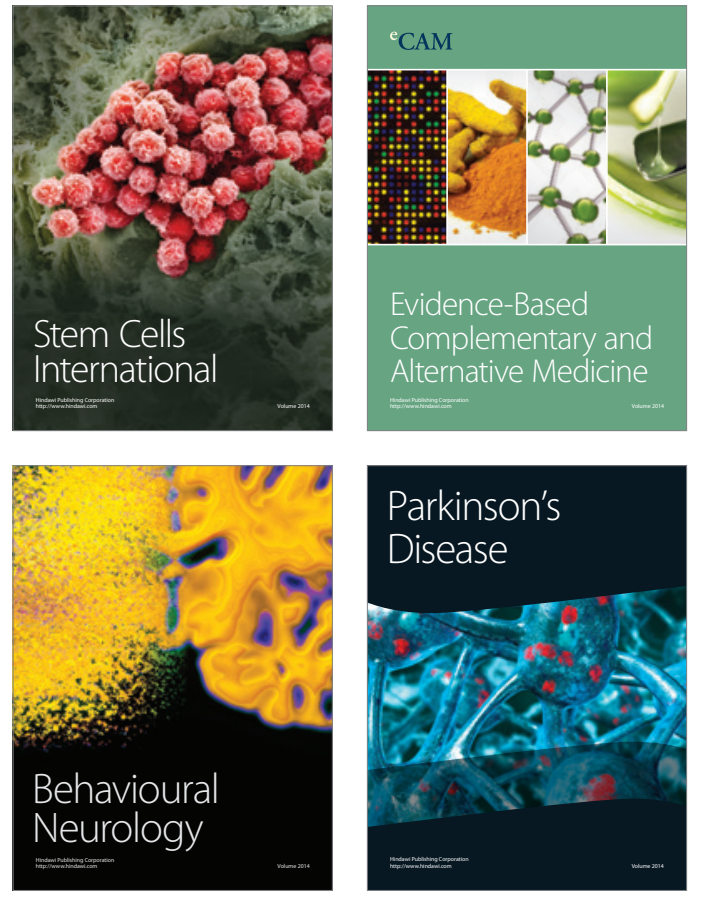
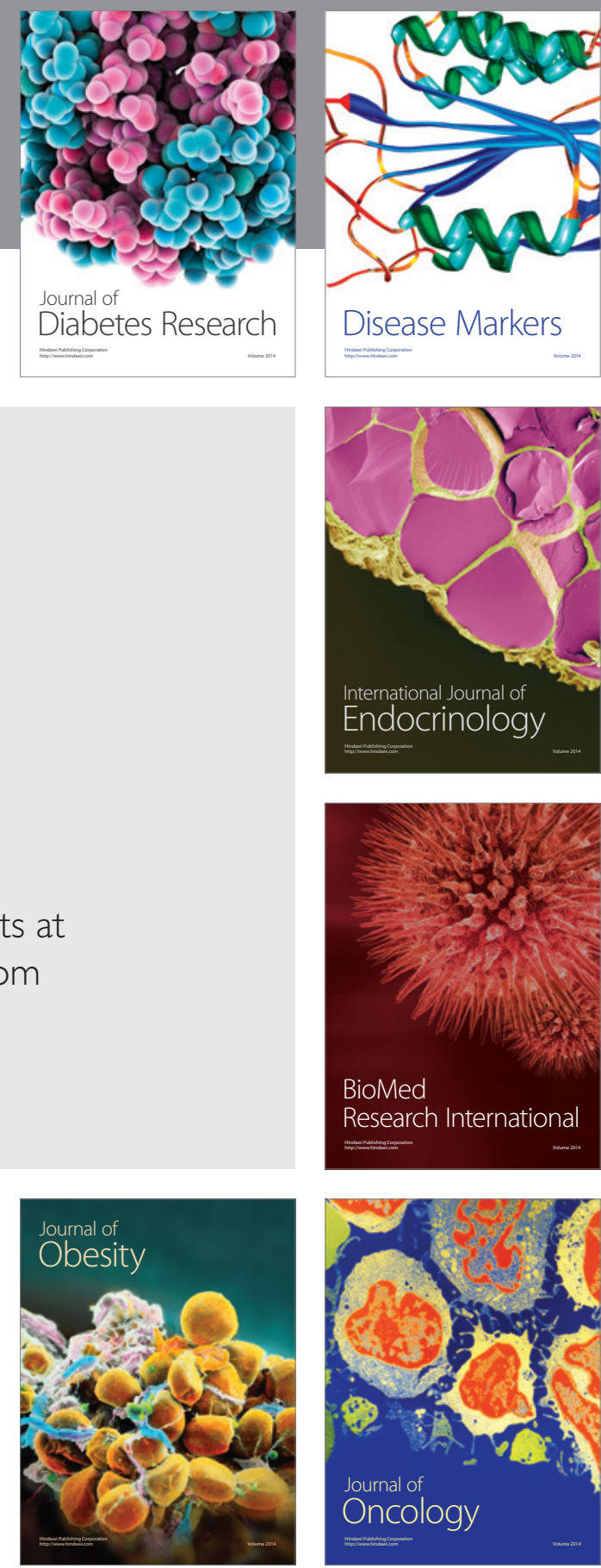

Disease Markers
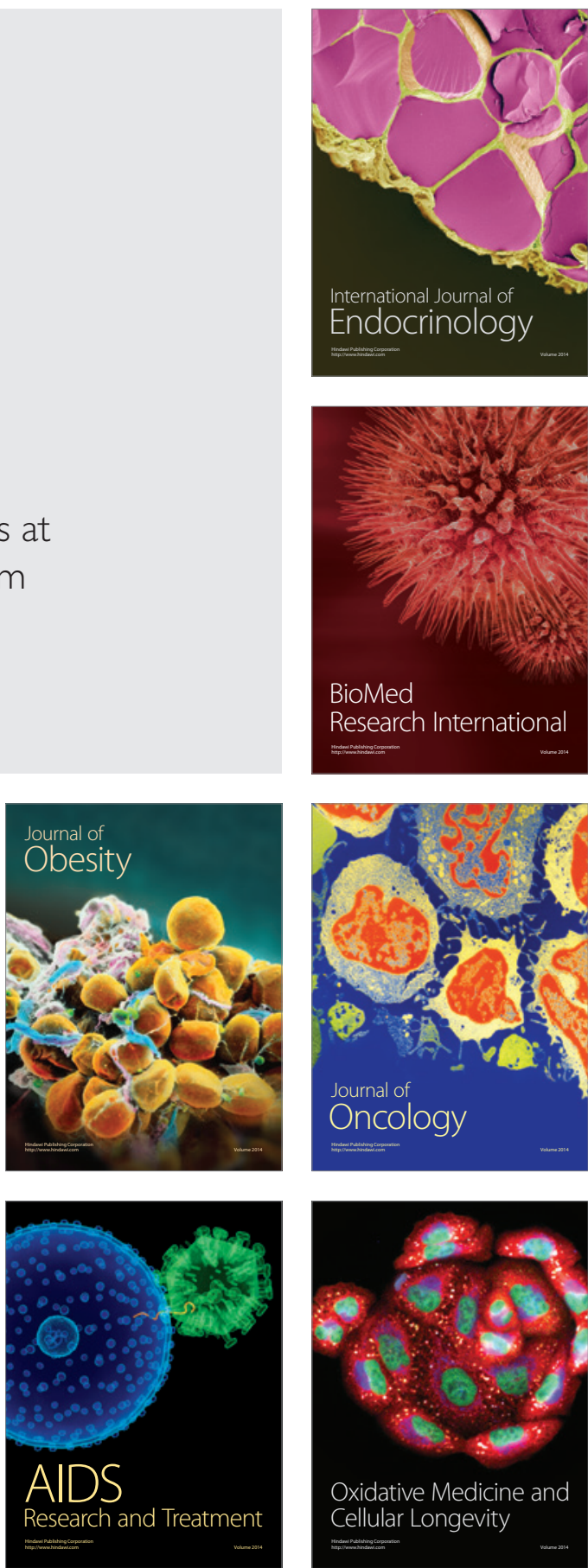